\title{
A PRIMAL-DUAL ACTIVE SET ALGORITHM FOR BILATERALLY CONTROL CONSTRAINED OPTIMAL CONTROL PROBLEMS
}

\author{
BY \\ MICHAEL HINTERMÜLLER \\ Department of Mathematics, Karl-Franzens University of Graz, Austria
}

\begin{abstract}
A generalized Moreau-Yosida based primal-dual active set algorithm for the solution of a representative class of bilaterally control constrained optimal control problems with boundary control is developed. The use of the generalized Moreau-Yosida approximation allows an efficient identification of the active and inactive sets at each iteration level. The method requires no step-size strategy and exhibits a finite termination property for the discretized problem class. In infinite as well as in finite dimensions a convergence analysis based on an augmented Lagrangian merit function is given. In a series of numerical tests the efficiency of the new algorithm is emphasized.
\end{abstract}

1. Introduction. In this paper we introduce and analyze an efficient algorithm for the numerical solution of the following bilaterally control constrained optimal control problem with boundary control:

$$
\begin{gathered}
\text { minimize } J(y, u)=\frac{1}{2} \int_{\Omega}\left(y-z_{d}\right)^{2} d x+\frac{\alpha}{2} \int_{\Gamma_{1}}\left(u-u_{d}\right)^{2} d s, \\
\text { subject to }-\Delta y+c y=g \text { in } \Omega, \quad \frac{\partial y}{\partial n}=u \text { on } \Gamma_{1}, \quad y=0 \text { on } \partial \Omega \backslash \Gamma_{1}=: \Gamma_{2} \\
\text { and } u \in U_{\text {ad }} \subset L^{2}\left(\Gamma_{1}\right),
\end{gathered}
$$

with a bounded domain $\Omega \subset \mathbb{R}^{d}, d \leq 3$, and $\partial \Omega=$ : $\Gamma$ its (sufficiently) smooth boundary. We assume that $\Gamma_{2} \subset \Gamma$ is closed and has positive $((d-1)$-dimensional) measure. Moreover, let $z_{d}, g \in L^{2}(\Omega), u_{d} \in L^{\infty}\left(\Gamma_{1}\right), \alpha>0, \bar{c} \geq c(x) \geq 0$ for all $x \in \Omega, \bar{c} \in \mathbb{R}$, $a, b \in L^{\infty}\left(\Gamma_{1}\right)$ with

$$
b(x)-a(x)>0 \text { for almost all } x \in \Gamma_{1} .
$$

Received July 1, 2000 and, in revised form, December 18, 2000.

2000 Mathematics Subject Classification. Primary 49J20, 49M29.

Key words and phrases. Active set, augmented Lagrangian, generalized Moreau-Yosida approximation, optimal control, primal-dual method.

The work was supported by the Fonds zur Förderung der wissenschaftlichen Forschung under "Spezialforschungsbereich Optimierung und Kontrolle", SFB03.

E-mail address: michael.hintermueller@kfunigraz.ac.at 
The set of admissible controls is given by $U_{\text {ad }}=\left\{u \in L^{2}\left(\Gamma_{1}\right) \mid a(x) \leq u(x) \leq b(x)\right.$ a.e. on $\left.\Gamma_{1}\right\}$. Equation (1.1b) will be referred to as the state equation.

The proposed algorithm and its analysis rely on the type of objective functional, governing state equation and the geometry of $U_{\mathrm{ad}}$, and it also applies to general radially unbounded convex quadratic objective functionals and state equations with any strictly elliptic second-order differential operator. However, for the sake of a clear presentation of the main ideas and a reduction of technicalities, we restrict ourselves to the specific representative (1.1) of the general problem class.

Our interest in this problem comes from the fact that it frequently occurs in applications either in its own right (see, e.g., [18]) or as a subproblem class in numerical schemes for efficiently solving nonlinear optimal control problems (see [13, 14, 16, 17, 25]). For example, consider the optimal control of a simplified Ginzburg-Landau model for superconductivity [23]:

$$
\begin{array}{ll}
\operatorname{minimize} & \frac{1}{2} \int_{\Omega}\left(y-z_{d}\right)^{2} d x+\frac{\alpha}{2} \int_{\Gamma_{1}} u^{2} d s \\
\text { subject to } & -\Delta y+y^{3}+y=g \text { in } \Omega, \quad \frac{\partial y}{\partial n}=u \text { on } \Gamma_{1}, \quad y=0 \text { on } \Gamma_{2} .
\end{array}
$$

If one uses a standard linearization technique for the treatment of the nonlinear state equation above, see, e.g., [6] where the SQP-framework is invoked, then one relies on an efficient solution of the linear-quadratic problem occurring in each iteration of the SQP-type algorithm. For our example, we obtain

$$
\begin{array}{ll}
\operatorname{minimize} & \frac{1}{2} \int_{\Omega}\left(y-z_{d}\right)^{2} d x+\frac{\alpha}{2} \int_{\Gamma_{1}} u^{2} d s \\
\text { subject to } & -\Delta y+\left(3 \bar{y}^{2}+1\right) y=\bar{g} \text { in } \Omega, \quad \frac{\partial y}{\partial n}=u \text { on } \Gamma_{1}, \quad y=0 \text { on } \Gamma_{2}
\end{array}
$$

with fixed $\bar{y}$ and appropriate $\bar{g}$. Additional control constraints of type (1.1c) yield the model problem (1.1).

Classical methods for solving the discretized model problem are either pure primal or dual methods; see, e.g., [8], [9]. Usually, in the case of box constraints, algorithms of gradient projection type-see, e.g., [3], [20]--outperform the classical methods. Modifications of projection type methods for large scale problems can be found in [21], or [19]. However, in the case of degenerate solutions, the convergence rate deteriorates. Only very recently Lin and Moré [19] proposed a trust region version of Newton's method that keeps fast local convergence even if the solution is degenerate. Another type of dual method that is applicable to large scale problems was proposed in [11] and [12]. In contrast to the gradient projection methods, it finds exact solutions. However, implementable versions require a suitable regularization. Finally, we mention interior point methods - see [26], [27], [28] and the references therein-which are very efficient in solving large convex quadratic minimization problems with linear constraints. Primal-dual path following variants of interior point methods utilize both the primal and the dual variables when following the central path towards the optimal solution. Typically, approximate solutions are found. Unfortunately, the convergence rate is only linear in the 
case of degenerate solutions. All above-mentioned methods were introduced and analyzed in finite dimensions. Some of them, like interior point methods, are intrinsically finite dimensional.

In [22], [7], and [16], an infinite-dimensional version of the projected Newton method in [3] is analyzed. Since the projected Newton method is designed for the minimization of a sufficiently smooth objective functional subject to box constraints, problems of type (1.1) must be transformed into a reduced form. For this purpose, let $\mathcal{S}$ denote the solution operator of the state equation, i.e., $y=\mathcal{S}(u)$. Then the reduced version of (1.1) is

$$
\begin{array}{ll}
\text { minimize } & \hat{J}(u):=\frac{1}{2} \int_{\Omega}\left(\mathcal{S}(u)-z_{d}\right)^{2} d x+\frac{1}{2} \int_{\Gamma_{1}}\left(u-u_{d}\right)^{2} d s \\
\text { subject to } \quad u \in U_{\mathrm{ad}} .
\end{array}
$$

Moreover, the methods in [22], [7], and [16] produce feasible iterates $u^{n}$, i.e., $u^{n} \in U_{\text {ad }}$ for all $n$, satisfying a descent property by a projected line search.

In contrast to these requirements and features, our algorithm is introduced and analyzed in infinite dimensions, it does not aim at the reduced problem (1.2), the iterates may be infeasible, and no line search is invoked. Moreover, it makes use of the primal and the dual variables at the same time. While the reduced problem approach is a matter of taste, the avoidance of the projected line search may speed up the performance. We also present the finite-dimensional counterpart of the proposed algorithm and analyze its convergence. We prove that it stops at the exact discrete solution after a finite number of iterations. Moreover, numerical tests confirm that our strategy incorporating primal and dual variables is very efficient and is not affected by degeneracy of optimal solutions.

The method we shall propose is based on a development due to [1]. The comprehensive numerical comparison in [4] with primal-dual path following interior point methods proves the efficiency of the algorithm in [1] for unilaterally constrained distributed control problems. In this paper, we extend the results in [1] in several ways: First, we consider the bilaterally constrained case, which is technically significantly more involved than the unilateral one. The conditions ensuring convergence must handle several degenerate situations as, for instance, change of components that are active on the upper constraint to infeasible components with respect to the lower bound. Secondly, we here consider boundary control whereas only distributed control was analyzed in [1]. As a result, stronger conditions on $\alpha$, and the operator norm $\left|A^{-1}\right|$ and $|\tau|$, with $A=-\Delta+c \cdot i d$ and $\tau$ the trace operator, have to be imposed. Moreover, we give results on the boundedness and convergence of the primal and dual iterates in infinite dimensions. Comparable results are not included in [1]. Also, a detailed discussion on several parameters involved in the convergence conditions are given.

The paper is organized as follows: In $\S 2$ we develop the first-order conditions for the model problem (1.1). Moreover, the basic tools for the algorithm are introduced. The infinite-dimensional algorithm is displayed in $\S 3$. The convergence analysis for the infinite-dimensional algorithm and a detailed discussion of the sufficient conditions for decrease of the proposed merit function are exhibited in $\S 4$. Section 5 contains the analysis of the finite-dimensional algorithm. A report on an excerpt of intensive numerical tests is given in $\S 6$. Finally, in $\S 7$ conclusions for our approach are drawn. 
2. Preliminaries. This section is devoted to the development of first-order conditions of the model problem (1.1). Moreover, we shall introduce all the tools needed for the definition of our algorithm.

Throughout this paper we shall use the following notation: the $L^{2}(\Omega)$ - and $L^{2}\left(\Gamma_{i}\right)$ inner products, $i=0,1,2$ with $\Gamma_{0}=\Gamma$, are denoted by $(\cdot, \cdot)_{\Omega}$ and $(\cdot, \cdot)_{\Gamma_{i}}$. By $|\cdot|_{\Omega}$ and $|\cdot| \Gamma_{i}$ the corresponding $L^{2}(\Omega)$ - and $L^{2}\left(\Gamma_{i}\right)$-norms are denoted. Frequently we shall use the duality pairing $(\cdot, \cdot)_{V^{*}, V}$ between a Hilbert space $V$ and its dual $V^{*}$. For $v \in H^{1}(\Omega)$, the zero-order trace $\tau: H^{1}(\Omega) \rightarrow L^{2}(\Gamma)$ is given by $\tau v$. Order relations like "max" or " $\leq$ " for elements of $L^{2}(\Omega)$ or $L^{2}\left(\Gamma_{i}\right)$ are understood in the pointwise almost everywhere sense.

For the following lemma, which establishes the existence of a unique solution of the state equation (1.1b), we define the closed subspace $V$ of $H^{1}(\Omega)$ by $V=\left\{v \in H^{1}(\Omega) \mid\right.$ $\left.\tau v_{\mid \Gamma_{2}}=0\right\}$. Here, $\tau v_{\mid \Gamma_{2}}$ denotes the restriction of the trace of $v$ on $\Gamma_{2}$. By $(\cdot, \cdot)_{V}=$ $(\cdot, \cdot)_{H^{1}(\Omega)}$ the inner product on $V$ is given. In order to make the paper self-contained, the proof of Lemma 2.1 is displayed in the appendix.

Lemma 2.1. Suppose $g \in L^{2}(\Omega), u \in L^{2}\left(\Gamma_{1}\right)$. Then the state equation (1.1b) admits a unique solution $y^{s} \in V$.

Proof. See Appendix A.

In the sequel we shall use $e: V \times L^{2}\left(\Gamma_{1}\right) \rightarrow V^{*}$ defined by

$$
(e(y, u), v)_{V^{*}, V}=(\nabla y, \nabla v)_{\Omega}+(c y, v)_{\Omega}-(g, v)_{\Omega}-\left(u, \tau v_{\mid \Gamma_{1}}\right)_{\Gamma_{1}} \quad \text { for all } v \in V .
$$

Then the weak form of the state equation (1.1b) becomes

$$
e(y, u)=0 \quad \text { in } V^{*} \text {. }
$$

By $e^{\prime}(y, u)$ we denote the gradient of $e(y, u)$ with respect to $y$ and $u$ :

$$
e^{\prime}(y, u)=\left(\begin{array}{l}
e_{y}(y, u) \\
e_{u}(y, u)
\end{array}\right) .
$$

Our next aim is to prove that $e^{\prime}$ is surjective, which is needed to guarantee the existence of a Lagrange multiplier in the first-order conditions.

LEMmA 2.2. The gradient $e^{\prime}(y, u)$ is surjective for all $(y, u) \in V \times L^{2}\left(\Gamma_{1}\right)$.

Proof. For any $(y, u) \in V \times L^{2}\left(\Gamma_{1}\right)$, consider

$$
\left(e^{\prime}(y, u)(\delta y, \delta u), v\right)_{V^{*}, V}=(\nabla \delta y, \nabla v)_{\Omega}+(c \delta y, v)_{\Omega}-\left(\delta u, \tau v_{\mid \Gamma_{1}}\right)_{\Gamma_{1}},
$$

with $(\delta y, \delta u) \in V \times L^{2}\left(\Gamma_{1}\right)$. In order to prove surjectivity, we arbitrarily fix $h \in V^{*}$. We then have to show that there exists $(\overline{\delta y}, \overline{\delta u}) \in V \times L^{2}\left(\Gamma_{1}\right)$ such that

$$
(\nabla \overline{\delta y}, \nabla v)_{\Omega}+(c \overline{\delta y}, v)_{\Omega}-\left(\overline{\delta u}, \tau v_{\mid \Gamma_{1}}\right)_{\Gamma_{1}}=h(v) \text { for all } v \in V .
$$

If we arbitrarily fix $\overline{\delta u} \in L^{2}\left(\Gamma_{1}\right)$, then for $\delta y \in V,(2.1)$ can be written as

$$
a(\delta y, v)=\hat{f}(v) \quad \text { for all } v \in V,
$$

with $\hat{f} \in V^{*}$ defined by $\hat{f}(v)=h(v)+\left(\overline{\delta u}, \tau v_{\mid \Gamma_{1}}\right)_{\Gamma_{1}}$. Since the bilinear form $a: V \times V \rightarrow$ $\mathbb{R}, a(w, v)=(\nabla w, \nabla v)_{\Omega}+(c w, v)_{\Omega}$, is $V$-elliptic and bounded, by the Lax-Milgram 
Theorem, the variational equality (2.2) admits a unique solution $\overline{\delta y} \in V$. This proves the surjectivity of $e^{\prime}(y, u)$ for all $(y, u) \in V \times L^{2}\left(\Gamma_{1}\right)$.

Now we are prepared to derive first-order optimality conditions. For this purpose we make use of the Lagrangian function defined by

$$
\tilde{\mathcal{L}}(y, u, p)=J(y, u)+(e(y, u), p)_{V^{*}, V}
$$

Let $\left(y^{*}, u^{*}\right)$ denote the optimal solution of (1.1). Uniqueness follows from the fact that $J$ is strictly convex in $u,(1.1 \mathrm{~b})$ admits a unique solution for every $u$, and $U_{\text {ad }}$ is convex. Next we define the active set at $\left(y^{*}, u^{*}\right)$ by

$$
\mathcal{A}^{*}=\mathcal{A}_{a}^{*} \cup \mathcal{A}_{b}^{*}
$$

with

$$
\mathcal{A}_{a}^{*}=\left\{x \in \Gamma_{1} \mid u(x)=a(x) \text { a.e. }\right\} \quad \text { and } \quad \mathcal{A}_{b}^{*}=\left\{x \in \Gamma_{1} \mid u(x)=b(x) \text { a.e. }\right\},
$$

and the inactive set at $\left(y^{*}, u^{*}\right)$ by

$$
\mathcal{I}^{*}=\Gamma_{1} \backslash \mathcal{A}^{*} .
$$

The first-order conditions are given by the next theorem.

ThEOREM 2.3. The unique solution $\left(y^{*}, u^{*}\right) \in V \times L^{2}\left(\Gamma_{1}\right)$ of $(1.1)$ is characterized by the existence of multipliers $p^{*} \in H^{1}(\Omega)$ and $\lambda^{*} \in L^{2}\left(\Gamma_{1}\right)$ such that

$$
\begin{aligned}
-\Delta p^{*}+c p^{*}+y^{*} & =z_{d} \quad \text { in } \Omega, \quad \frac{\partial p^{*}}{\partial n}=0 \quad \text { on } \Gamma_{1}, \quad p^{*}=0 \quad \text { on } \Gamma_{2}, \\
\alpha u^{*}+\lambda^{*}-\tau p_{\mid \Gamma_{1}}^{*} & =\alpha u_{d} \quad \text { on } \Gamma_{1}, \\
e\left(y^{*}, u^{*}\right) & =0, \quad u^{*} \in U_{\mathrm{ad}}, \\
\lambda_{\mid \mathcal{A}_{a}^{*}}^{*} & \leq 0, \quad \lambda_{\mid \mathcal{A}_{b}^{*}}^{*} \geq 0, \quad \lambda_{\mid \mathcal{I}^{*}}^{*}=0,
\end{aligned}
$$

with $\lambda_{\mid S}^{*}$ denoting the multiplier on a subset $S$ of $\Gamma_{1}$.

Proof. First observe that Lemma 2.2 ensures the existence of $p^{*} \in V$ such that

$$
\tilde{\mathcal{L}}_{y}\left(y^{*}, u^{*}, p^{*}\right)=0 \quad \text { and } \quad e\left(y^{*}, u^{*}\right)=0 .
$$

More precisely, we have

$$
\tilde{\mathcal{L}}(y, u, p)=J(y, u)+(\nabla y, \nabla p)_{\Omega}+(c y, p)_{\Omega}-(g, p)_{\Omega}-\left(u, \tau p_{\mid \Gamma_{1}}\right)_{\Gamma_{1}},
$$

yielding

$$
\begin{aligned}
\tilde{\mathcal{L}}_{y}\left(y^{*}, u^{*}, p^{*}\right) \delta y & =\left(y^{*}-z_{d}, \delta y\right)_{\Omega}+\left(\nabla p^{*}, \nabla \delta y\right)_{\Omega}+\left(c p^{*}, \delta y\right)_{\Omega} \\
& =\left(y^{*}-z_{d}, \delta y\right)_{\Omega}+\left(-\Delta p^{*}, \delta y\right)_{\Omega}+\left(c p^{*}, \delta y\right)_{\Omega}
\end{aligned}
$$

for $\delta y \in V$ and $\frac{\partial p^{*}}{\partial n}=0$ on $\Gamma_{1}, \tau p_{\mid \Gamma_{2}}^{*}=0$. This yields (2.3a). Condition (2.3c), which denotes feasibility, is trivially satisfied.

For optimality in $u$ the following variational inequality has to be satisfied:

$$
\tilde{\mathcal{L}}_{u}\left(y^{*}, u^{*}, p^{*}\right)\left(u-u^{*}\right) \geq 0 \text { for all } u \in U_{\mathrm{ad}} .
$$

This is equivalent to

$$
\left(-\lambda^{*}, u-u^{*}\right)_{\Gamma_{1}}:=\left(\alpha\left(u^{*}-u_{d}\right)-\tau p_{\mid \Gamma_{1}}^{*}, u-u^{*}\right)_{\Gamma_{1}} \geq 0 \text { for all } u \in U_{\mathrm{ad}} .
$$


There are three distinct cases:

(i) Consider $\mathcal{A}_{a}^{*}$, i.e., $u^{*}=a$. Define

$$
\mathcal{A}_{a}^{+}=\left\{x \in \Gamma_{1} \mid\left(u^{*}(x)=a(x)\right) \wedge\left(\lambda^{*}(x)>0\right)\right\}, \quad \mathcal{A}_{a}^{+^{\imath}}=\left\{x \in \mathcal{A}_{a}^{+} \mid \lambda^{*}(x)>\frac{1}{l}\right\}
$$

and $\mathcal{C}_{a}^{l}=\left\{x \in \mathcal{A}_{a}^{+l} \mid b(x)-a(x)>\frac{1}{l}\right\}$. Now assume that $\mathcal{A}_{a}^{+}$has positive measure $\operatorname{meas}\left(\mathcal{A}_{a}^{+}\right)>\epsilon>0$. Since meas $\left\{x \in \Gamma_{1} \mid a(x)=b(x)\right\}=0$ and $\mathcal{A}_{a}^{+{ }^{l}} \uparrow \mathcal{A}_{a}^{+}$, we have meas $\left(\mathcal{C}_{a}^{l}\right)>0$ for sufficiently large $l$ and $\mathcal{C}_{a}^{l} \uparrow \mathcal{A}_{a}^{+}$. From the lower continuity of meas, we deduce that there exists $\ell>0$ such that meas $\left(\mathcal{C}_{a}^{\ell}\right) \geq \epsilon$. Next define $\delta^{+}=\chi_{\mathcal{C}_{a}^{\ell}}(b-a)$, which yields $a \leq u^{*}+s \delta^{+} \leq b$ for $0 \leq s \leq 1$ ( $\chi_{S}$ denotes the characteristic function of a set $\left.S \subset \Gamma_{1}\right)$. Consider the directional derivative

$$
\left.\frac{d \tilde{\mathcal{L}}\left(y^{*}, u^{*}+s \delta^{+}, p^{*}\right)}{d s}\right|_{s=0}=\left(\alpha\left(u^{*}-u_{d}\right)-\tau p_{\mid \Gamma_{1}}^{*}, \delta^{+}\right)_{\Gamma_{1}}=\left(-\lambda^{*}, \delta^{+}\right)_{\Gamma_{1}}<-\frac{\epsilon}{\ell^{2}}<0 .
$$

This contradicts the optimality of $u^{*}$. Hence, we must have meas $\left(\mathcal{A}_{a}^{+}\right)=0$.

(ii) One can prove in the same way as in (i) that meas $\left(\mathcal{A}_{b}^{-}\right)=0$ with

$$
\mathcal{A}_{b}^{-}=\left\{x \in \Gamma_{1} \mid\left(u^{*}(x)=b(x)\right) \wedge\left(\lambda^{*}(x)<0\right)\right\} .
$$

(iii) Finally, consider $\mathcal{I}^{*}$, i.e., $a<u^{*}<b$, and define

$$
\mathcal{I}=\left\{x \in \Gamma_{1} \mid\left(a(x)<u^{*}(x)<b(x)\right) \wedge\left(\lambda^{*}(x) \neq 0\right)\right\}
$$

and for $l \in \mathbb{N}$

$$
\mathcal{I}^{l}=\left\{x \in \mathcal{I} \mid\left(a(x)+\frac{1}{l} \leq u^{*}(x) \leq b(x)-\frac{1}{l}\right) \wedge\left(\left|\lambda^{*}(x)\right| \geq \frac{1}{l}\right)\right\} .
$$

Assume that $\operatorname{meas}(\mathcal{I})>\epsilon>0$. Since $\operatorname{meas}\left\{x \in \Gamma_{1} \mid a(x)=b(x)\right\}=0$, we obtain meas $\left(\mathcal{I}^{l}\right)>0$ for sufficiently large $l$. From $\mathcal{I}^{l} \uparrow \mathcal{I}$ and the lower continuity of meas, we deduce that there exists $\ell>0$ such that meas $\left(\mathcal{I}^{\ell}\right) \geq \epsilon$. Define $\delta=\chi_{\mathcal{I}^{\ell}}\left(\lambda^{*}(x) /\left|\lambda^{*}(x)\right|\right)$ implying $a \leq u^{*}+s \delta \leq b$ for $0 \leq s \leq \frac{1}{\ell}$, and

$$
\left.\frac{d \tilde{\mathcal{L}}\left(y^{*}, u^{*}+s \delta, p^{*}\right)}{d s}\right|_{s=0}=\left(-\lambda^{*}, \delta\right)_{\Gamma_{1}} \leq-\frac{\epsilon}{\ell}<0 .
$$

This contradicts the optimality of $u^{*}$. Thus, there must be meas $(\mathcal{I})=0$.

Note that for the above first-order conditions, it suffices to consider one multiplier for both inequalities characterizing feasibility. The benefit of this fact will become clear in $\S 5$ where the discretized problem class is considered. The restriction to one multiplier for both inequalities reduces the number of variables and consequently the amount of memory needed by an implementable algorithm.

Before we state the algorithm, we make use of a result from convex analysis. Based on the generalized Moreau-Yosida approximation of the indicator function of the set of admissible controls denoted by $U_{\text {ad }}$ one can replace $u^{*} \in U_{\text {ad }}$ and condition (2.3d) by

$$
\lambda^{*}=\sigma\left[u^{*}+\sigma^{-1} \lambda^{*}-\pi_{U_{\mathrm{ad}}}\left(u^{*}+\sigma^{-1} \lambda^{*}\right)\right],
$$


for $\sigma>0$, where

$$
\pi_{U_{\mathrm{ad}}}(z)= \begin{cases}a & \text { if } z<a \\ z & \text { if } a \leq z \leq b \\ b & \text { if } z>b\end{cases}
$$

denotes the Hilbert-space projection onto $U_{\mathrm{ad}}$. For more details we refer to [2], [15].

3. The algorithm. In this section we present the generalized Moreau-Yosida-based active set algorithm. We make use of the characterization (2.5) of the optimal Lagrange multiplier. Let superscript $n$ denote the actual iteration level. Then we define the $a$ active and $b$-active sets of the $n$th iteration by

$$
\begin{aligned}
& \mathcal{A}_{a}^{n}=\left\{x \in \Gamma_{1} \mid u^{n-1}(x)+\sigma^{-1} \lambda^{n-1}(x)<a(x) \text { a.e. }\right\} \\
& \mathcal{A}_{b}^{n}=\left\{x \in \Gamma_{1} \mid u^{n-1}(x)+\sigma^{-1} \lambda^{n-1}(x)>b(x) \text { a.e. }\right\}
\end{aligned}
$$

and $\mathcal{A}^{n}=\mathcal{A}_{a}^{n} \cup \mathcal{A}_{b}^{n}$ and $\mathcal{I}^{n}=\Gamma_{1} \backslash \mathcal{A}^{n}$. Note that the definition of $\mathcal{A}^{n}$ and $\mathcal{I}^{n}$ involves both the primal variable $u$ and the dual variable $\lambda$. This strategy turns out to be very efficient in practice (see $\S 6$ ). In the algorithm below, we use the identification $\mathcal{A}^{n}=\mathcal{A}^{n-1}$, which is understood in the sense

$$
\mathcal{A}_{a}^{n}=\mathcal{A}_{a}^{n-1} \quad \text { and } \quad \mathcal{A}_{b}^{n}=\mathcal{A}_{b}^{n-1} .
$$

\section{Algorithm AS}

begin

$\left(y^{o}, u^{o}, p^{o}, \lambda^{o}\right) \leftarrow$ initialization $\left(\Omega, \Gamma, \Gamma_{1}, c, \alpha, z_{d}, u_{d}, g\right)$

$n \leftarrow 0$

end

while $\left(\mathcal{A}^{n} \neq \mathcal{A}^{n-1}\right)$ or $(n<2)$

$$
\begin{aligned}
\mathcal{A}_{a}^{n} & \leftarrow\left\{x \in \Gamma_{1} \mid u^{n-1}(x)+\sigma^{-1} \lambda^{n-1}(x)<a(x) \text { a.e. }\right\} \\
\mathcal{A}_{b}^{n} & \leftarrow\left\{x \in \Gamma_{1} \mid u^{n-1}(x)+\sigma^{-1} \lambda^{n-1}(x)>b(x) \text { a.e. }\right\} \\
\mathcal{A}^{n} & \leftarrow \mathcal{A}_{a}^{n} \cup \mathcal{A}_{b}^{n}, \mathcal{I}^{n} \leftarrow \Gamma_{1} \backslash \mathcal{A}^{n} \\
u_{\mid \mathcal{A}_{a}^{n}}^{n} & \leftarrow a_{\mid \mathcal{A}_{a}^{n}}, u_{\mid \mathcal{A}_{b}^{n}}^{n} \leftarrow b_{\mid \mathcal{A}_{b}^{n}}, \lambda_{\mid \mathcal{I}^{n}}^{n} \leftarrow 0 .
\end{aligned}
$$

Determine $y^{n}, p^{n}$ and $u_{\mid \mathcal{I}^{n}}^{n}, \lambda_{\mid \mathcal{A}^{n}}^{n}$ such that $\left(y^{n}, u^{n}, p^{n}, \lambda^{n}\right)$ satisfies (3.2a)-(3.2c). end

Different initialization routines may be used. We use the following scheme, which is intended to obtain a feasible start-up configuration. In parentheses an alternative is denoted.

\section{initialization}

Input: $\Omega, \Gamma, \Gamma_{1}, c, \alpha, z_{d}, u_{d}, g$.

Output: $y^{o}, u^{o}, p^{o}, \lambda^{o}$.

begin

$u^{o} \leftarrow a .\left(u^{o} \leftarrow b\right)$

Obtain $y^{o}, p^{o}$ as solution to (3.2a) and (3.2c).

$\lambda^{o} \leftarrow \min \left\{0, \tau p_{\mid \Gamma_{1}}^{o}+\alpha\left(u_{d}-u^{o}\right)\right\} .\left(\lambda^{o} \leftarrow \max \left\{0, \tau p_{\mid \Gamma_{1}}^{o}+\alpha\left(u_{d}-u^{o}\right)\right\}\right)$

\section{end}


The next result is a justification of the stopping rule for Algorithm AS.

Lemma 3.1. If there exists an iteration level $n$ such that $\mathcal{A}^{n}=\mathcal{A}^{n+1}$, then Algorithm AS stops and the last iterate satisfies

$$
\begin{aligned}
& -\Delta p^{n}+c p^{n}+y^{n}=z_{d} \quad \text { in } \Omega, \quad \frac{\partial p^{n}}{\partial n}=0 \quad \text { on } \Gamma_{1}, \quad p^{n}=0 \quad \text { on } \Gamma_{2} \\
& \alpha u^{n}+\lambda^{n}-\tau p_{\mid \Gamma_{1}}^{n}=\alpha u_{d} \quad \text { on } \Gamma_{1} \\
& -\Delta y^{n}+c y^{n}=g \quad \text { in } \Omega, \quad \frac{\partial y^{n}}{\partial n}=u^{n} \quad \text { on } \Gamma_{1}, \quad y^{n}=0 \quad \text { on } \Gamma_{2}, \\
& u^{n} \in U_{\mathrm{ad}} \\
& \lambda_{\mid \mathcal{A}_{a}^{n}}^{n} \leq 0, \quad \lambda_{\mid \mathcal{A}_{b}^{n}}^{n} \geq 0, \quad \lambda_{\mid \mathcal{I}^{n}}^{n}=0 .
\end{aligned}
$$

Proof. By construction of the iteration sequence, the conditions $(3.2 \mathrm{a})-(3.2 \mathrm{c})$ are satisfied. Therefore, we only have to concentrate on the feasibility of the control and the sign of the corresponding Lagrange multiplier. For this purpose, consider first $\mathcal{I}^{n+1}$. Here we have $\lambda^{n}=0$ by construction. The set $\mathcal{I}^{n+1}$ is defined as the set of $x \in \Gamma_{1}$ such that

$$
a(x) \leq u^{n}(x)+\sigma^{-1} \lambda^{n}(x) \leq b(x),
$$

yielding $a \leq u^{n} \leq b$ in $\mathcal{I}^{n}$. In $\mathcal{A}_{a}^{n}$ we have $u^{n}=a$. The way in which $\mathcal{A}_{a}^{n+1}$ is defined yields $\lambda^{n}<0$ in $\mathcal{A}_{a}^{n}$. Analogous arguments yield $u^{n}=b$ and $\lambda^{n}>0$ in $\mathcal{A}_{b}^{n}$.

4. Convergence analysis. Our convergence analysis is based on an appropriately chosen merit function. In fact, we will use the modified augmented Lagrangian functional $L: V \times L^{2}\left(\Gamma_{1}\right) \times L^{2}\left(\Gamma_{1}\right) \rightarrow \mathbb{R}$ defined by

$$
\begin{aligned}
L\left(y^{n}, u^{n}, \lambda^{n}\right)=J\left(y^{n}, u^{n}\right)+\frac{1}{2 \sigma}\left[\chi_{S_{a}^{n}}(\mid\right. & \left.\left.\left(\sigma\left(a-u^{n}\right)-\lambda_{-}^{n}\right)_{+}\right|_{\Gamma_{1}} ^{2}-\left|\lambda_{-}^{n}\right|_{\Gamma_{1}}^{2}\right) \\
& \left.+\chi_{S_{b}^{n}}\left(\left|\left(\sigma\left(u^{n}-b\right)+\lambda_{+}^{n}\right)_{+}\right|_{\Gamma_{1}}^{2}-\left|\lambda_{+}^{n}\right|_{\Gamma_{1}}^{2}\right)\right],
\end{aligned}
$$

where $(\cdot)_{+}=\max \{0, \cdot\}$, and further

$$
S_{a}^{n}=\mathcal{I}^{n} \cup \mathcal{A}_{a}^{n} \quad \text { and } \quad S_{b}^{n}=\mathcal{I}^{n} \cup \mathcal{A}_{b}^{n} .
$$

By $\lambda_{+}$and $\lambda_{-}$we denote the positive and negative part of the multiplier $\lambda$, which are defined by

$$
\lambda_{+}=\max \{0, \lambda\} \quad \text { and } \quad \lambda_{-}=\min \{0, \lambda\} .
$$

We will prove that under the condition on $\alpha$ and $\sigma$ given by

$$
\rho+\alpha \leq \sigma<\alpha+\frac{\alpha^{2}}{C^{2}}-\frac{\alpha^{2}}{\rho} \quad \text { and } \quad(\sigma-\alpha)\left|u^{n-1}-u^{n}\right|_{\mathcal{P}^{n}}^{2}-\sigma|a-b|_{\mathcal{P}^{n}}^{2} \leq 0
$$

for some $\rho>0$, the modified augmented Lagrangian satisfies

$$
L\left(y^{n}, u^{n}, \lambda^{n}\right)-L\left(y^{n-1}, u^{n-1}, \lambda^{n-1}\right)<0 .
$$

The set $\mathcal{P}^{n}$ is defined by (4.3).

Before we can prove the above convergence assertion, we have to establish a few auxiliary results. The first result relates the difference of objective functionals to the primal variables $(y, u)$ and the dual variable $\lambda$. 
Lemma 4.1. Suppose $(y, u) \in V \times L^{2}\left(\Gamma_{1}\right)$ satisfies

$$
-\Delta y+c y=g \quad \text { in } \Omega, \quad \frac{\partial y}{\partial n}=u \quad \text { on } \Gamma_{1}, \quad y=0 \quad \text { on } \Gamma_{2} .
$$

Then for all iteration levels $n \geq 1$ we have

$$
J\left(y^{n}, u^{n}\right)-J(y, u)=-\frac{1}{2}\left|y-y^{n}\right|_{\Omega}^{2}-\frac{\alpha}{2}\left|u-u^{n}\right|_{\Gamma_{1}}^{2}+\left(u-u^{n}, \lambda^{n}\right)_{\mathcal{A}^{n}} .
$$

Proof. The construction of the iteration sequence of Algorithm AS and $|p|^{2}-|q|^{2}=$ $-|p-q|^{2}+2(p-q, p)$ yield

$$
\begin{aligned}
& J\left(y^{n}, u^{n}\right)-J(y, u) \\
&=- \frac{1}{2}\left|y-y^{n}\right|_{\Omega}^{2}-\frac{\alpha}{2}\left|u-u^{n}\right|_{\Gamma_{1}}^{2}+\left(y^{n}-y, y^{n}-z_{d}\right)_{\Omega}+\alpha\left(u^{n}-u, u^{n}-u_{d}\right)_{\Gamma_{1}} \\
&=-\frac{1}{2}\left|y-y^{n}\right|_{\Omega}^{2}-\frac{\alpha}{2}\left|u-u^{n}\right|_{\Gamma_{1}}^{2}+\left(y^{n}-y, \Delta p^{n}-c p^{n}\right)_{\Omega}+\alpha\left(u^{n}-u, u^{n}-u_{d}\right)_{\Gamma_{1}} \\
&=-\frac{1}{2}\left|y-y^{n}\right|_{\Omega}^{2}-\frac{\alpha}{2}\left|u-u^{n}\right|_{\Gamma_{1}}^{2}+\left(\Delta\left(y^{n}-y\right)-c\left(y^{n}-y\right), p^{n}\right)_{\Omega} \\
&+\left(\frac{\partial\left(y-y^{n}\right)}{\partial n}, \tau p_{\mid \Gamma_{1}}^{n}\right)_{\Gamma_{1}}+\alpha\left(u^{n}-u, u^{n}-u_{d}\right)_{\Gamma_{1}} \\
&=-\frac{1}{2}\left|y-y^{n}\right|_{\Omega}^{2}-\frac{\alpha}{2}\left|u-u^{n}\right|_{\Gamma_{1}}^{2}+\left(u^{n}-u, \alpha\left(u^{n}-u_{d}\right)-\tau p_{\mid \Gamma_{1}}^{n}\right)_{\Gamma_{1}} \\
&=-\frac{1}{2}\left|y-y^{n}\right|_{\Omega}^{2}-\frac{\alpha}{2}\left|u-u^{n}\right|_{\Gamma_{1}}^{2}+\left(u-u^{n}, \lambda^{n}\right)_{\mathcal{A}^{n}},
\end{aligned}
$$

where the last equality comes from $\lambda_{\mid \mathcal{I}^{n}}^{n}=0$, completing the proof.

As we consider the difference of the objective functionals of two successive iterates of Algorithm AS, then we obtain the immediate corollary of Lemma 4.1.

Corollary 4.2. Let $\left(y^{i}, u^{i}, \lambda^{i}\right), i=n-1, n$, denote two successive iterates of Algorithm AS. Then

$$
J\left(y^{n}, u^{n}\right)-J\left(y^{n-1}, u^{n-1}\right)=-\frac{1}{2}\left|y^{n-1}-y^{n}\right|_{\Omega}^{2}-\frac{\alpha}{2}\left|u^{n-1}-u^{n}\right|_{\Gamma_{1}}^{2}+\left(u^{n-1}-u^{n}, \lambda^{n}\right)_{\mathcal{A}_{*}^{n}},
$$

with $\mathcal{A}_{*}^{n}$ the subset of $\mathcal{A}^{n}$ defined by

$$
\mathcal{A}_{*}^{n}=\left(\mathcal{A}_{a}^{n} \backslash \mathcal{A}_{a}^{n-1}\right) \cup\left(\mathcal{A}_{b}^{n} \backslash \mathcal{A}_{b}^{n-1}\right) .
$$

Proof. The assertion immediately follows when considering $u^{n-1}-u^{n}=0$ on $\left(\mathcal{A}_{a}^{n} \cap\right.$ $\left.\mathcal{A}_{a}^{n-1}\right) \cup\left(\mathcal{A}_{b}^{n} \cap \mathcal{A}_{b}^{n-1}\right)$ and $\mathcal{A}_{*}^{n}=\mathcal{A}^{n} \backslash\left(\left(\mathcal{A}_{a}^{n} \cap \mathcal{A}_{a}^{n-1}\right) \cup\left(\mathcal{A}_{b}^{n} \cap \mathcal{A}_{b}^{n-1}\right)\right)$.

The following lemma estimates the term under brackets in the definition (4.1) of the modified augmented Lagrangian function. For convenience we use

$$
\begin{aligned}
q^{n}(x)= & \chi_{S_{a}^{n}}(x)\left(\left|\left(\sigma\left(a(x)-u^{n}(x)\right)-\lambda_{-}^{n}(x)\right)_{+}\right|^{2}-\left|\lambda_{-}^{n}(x)\right|^{2}\right) \\
& +\chi_{S_{b}^{n}}(x)\left(\left|\left(\sigma\left(u^{n}(x)-b(x)\right)+\lambda_{+}^{n}(x)\right)_{+}\right|^{2}-\left|\lambda_{+}^{n}(x)\right|^{2}\right)
\end{aligned}
$$

for $x \in \Gamma_{1}$. Moreover, we define

$$
d^{n}(x)=q^{n}(x)-q^{n-1}(x) .
$$


The analysis in the proof of the following Lemma 4.3 will show that the set $\mathcal{I}^{n}$ especially is of interest in view of its history. For this purpose we use the following splitting of $\Gamma_{1}$ :

$$
\begin{aligned}
& \mathcal{I}_{*}^{n}=\left(\mathcal{I}^{n-1} \cap \mathcal{I}^{n}\right) \cup\left(\mathcal{A}_{a}^{n-1} \cap \mathcal{I}_{-}^{n}\right) \cup\left(\mathcal{A}_{b}^{n-1} \cap \mathcal{I}_{+}^{n}\right), \\
& \mathcal{F}_{a}^{n}=\mathcal{A}_{a}^{n-1} \cap\left\{x \in \mathcal{I}^{n} \mid a(x) \leq u^{n}(x) \leq b(x) \text { a.e. }\right\}, \\
& \mathcal{F}_{b}^{n}=\mathcal{A}_{b}^{n-1} \cap\left\{x \in \mathcal{I}^{n} \mid a(x) \leq u^{n}(x) \leq b(x) \text { a.e. }\right\}, \\
& \mathcal{P}_{a}^{n}=\mathcal{A}_{a}^{n-1} \cap \mathcal{I}_{+}^{n}, \\
& \mathcal{P}_{b}^{n}=\mathcal{A}_{b}^{n-1} \cap \mathcal{I}_{-}^{n},
\end{aligned}
$$

where $\mathcal{I}_{-}^{n}=\left\{x \in \mathcal{I}^{n} \mid u^{n}(x)<a(x)\right.$ a.e. $\}$ and $\mathcal{I}_{+}^{n}=\left\{x \in \mathcal{I}^{n} \mid u^{n}(x)>b(x)\right.$ a.e. $\}$. For later use we shall define $\mathcal{P}^{n}=\mathcal{P}_{a}^{n} \cup \mathcal{P}_{b}^{n}$. Let us give a few comments on this splitting.

First of all observe that

$$
\mathcal{I}_{*}^{n} \cup \mathcal{F}_{a}^{n} \cup \mathcal{F}_{b}^{n} \cup \mathcal{P}^{n}=\mathcal{I}^{n}
$$

The splitting basically serves as a monitor for the movement of the respective sets from one iteration level to the next.

In contrast to the unilaterally constrained problem, i.e., the problem with either $u \leq b$ or $u \geq a$ defining the set of admissible controls, the sets $\mathcal{P}_{a}^{n}$ and $\mathcal{P}_{b}^{n}$ have to be taken into account. Details will be given below. But for convenience let us give a schematic graph (Fig. 1) for the meaning of $\mathcal{P}_{a}^{n}$. For the sake of simplicity we assume that $\mathcal{A}_{b}^{n-1}=\varnothing$ in the following exposition: First consider the set of points $x \in \Gamma_{1}$ where the corresponding control $u^{n-1}$ of iteration level $n-1$ is active at the lower bound $a$, but the Lagrange multiplier indicates that $u^{n-1}$ should be inactive, i.e., $\lambda^{n-1} \geq 0$. This set corresponds to the left part of $\mathcal{A}_{a}^{n-1}$ in Fig. 1. Consequently this part becomes a subset of $\mathcal{I}^{n}$ in iteration level $n$. Now, $\mathcal{P}_{a}^{n}$ contains all $x \in \mathcal{A}_{a}^{n-1} \cap \mathcal{I}^{n}$ whose corresponding $u^{n}(x)$ exceeds the upper bound $b(x)$ in the almost everywhere sense. The second case, i.e. $\mathcal{P}_{b}^{n}$, is an analogue.

Now, we are well prepared to prove the following lemma, which gives estimates for $d^{n}(x)$ for all iteration levels $n$.

Lemma 4.3. Let $\left(y^{i}, u^{i}, \lambda^{i}\right), i=n, n-1$ denote two successive iterates of Algorithm AS. Then

$$
\begin{aligned}
& d^{n}(x) \leq \sigma^{2}\left|u^{n-1}(x)-u^{n}(x)\right|^{2} \quad \text { for a.a. } x \in \mathcal{I}_{*}^{n}, \\
& d^{n}(x)=0 \text { for a.a. } x \in \mathcal{F}_{a}^{n} \cup \mathcal{F}_{b}^{n} \cup\left(\mathcal{A}^{n-1} \cap \mathcal{A}^{n}\right), \\
& d^{n}(x)<\sigma^{2}\left(\left|u^{n-1}(x)-u^{n}(x)\right|^{2}-|a(x)-b(x)|^{2}\right) \quad \text { for a.a. } x \in \mathcal{P}^{n}, \\
& d^{n}(x) \leq-\sigma^{2}\left|u^{n-1}(x)-u^{n}(x)\right|^{2} \quad \text { for a.a. } x \in \mathcal{T}^{n-1},
\end{aligned}
$$

with $\mathcal{T}^{n-1}=\mathcal{I}^{n-1} \cap \mathcal{A}^{n}$

Proof. The proof considers the different subsets of iteration levels $n$ and $n-1$ and finally extracts our desired result. For convenience we skip the argument $x$ and argue for almost all (a.a.) $x$ in the respective sets. 


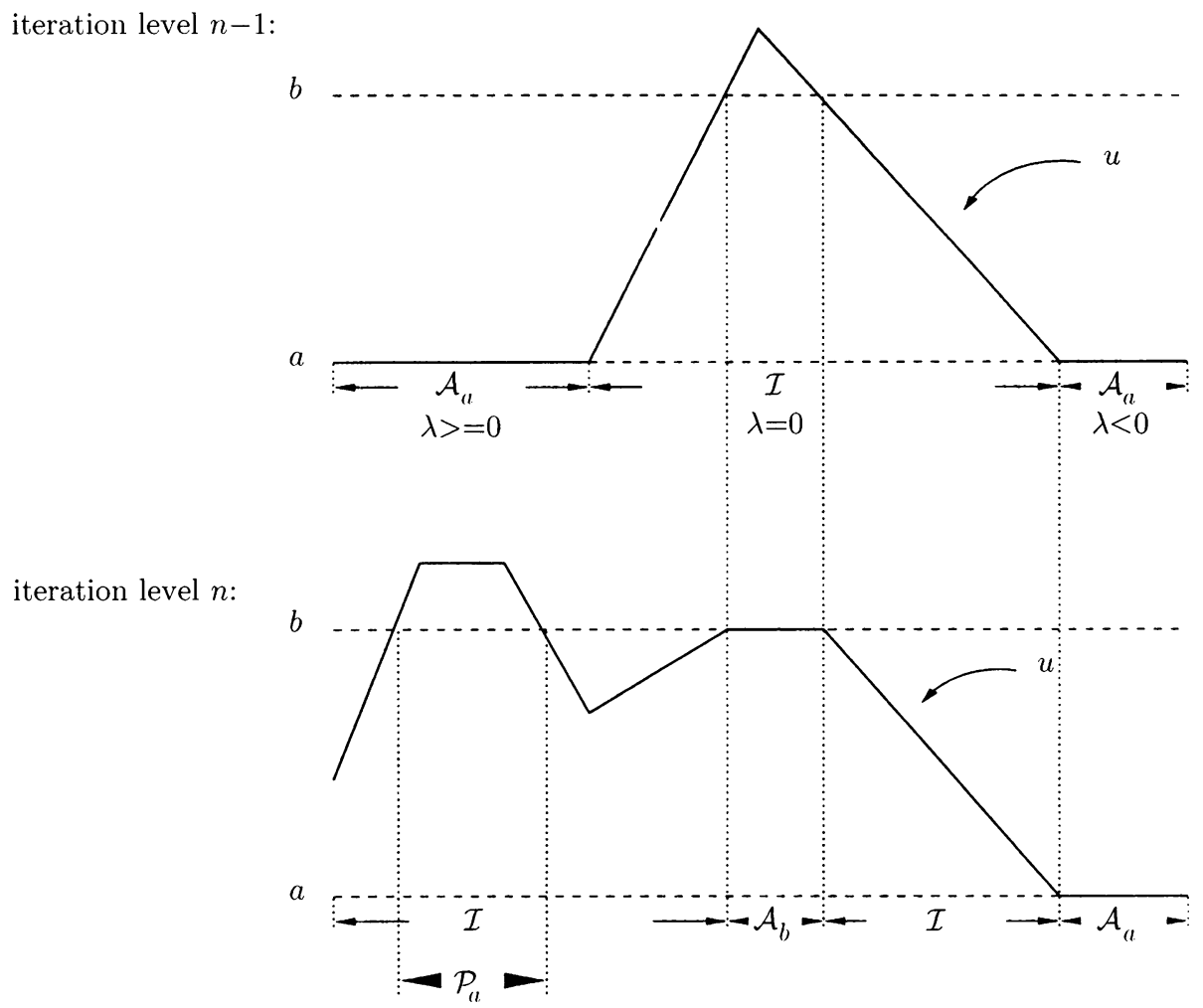

FIG. 1. Study of $\mathcal{P}_{a}^{n}$

(i) $\mathcal{I}^{n-1} \cap \mathcal{I}^{n}$ : By construction $\lambda^{n-1}=\lambda^{n}=0$ and $a \leq u^{n-1} \leq b$ by the way in which $\mathcal{I}^{n}$ is defined. Hence

$$
\begin{aligned}
d^{n} & \left.=\left|\left(\sigma\left(u^{n}-b\right)\right)_{+}\right|^{2}+\left|\left(\sigma\left(a-u^{n}\right)\right)_{+}\right|^{2} \leq\left.\sigma^{2}\left(\mid\left(u^{n}-u^{n-1}\right)\right)_{+}\right|^{2}+\left|\left(u^{n-1}-u^{n}\right)_{+}\right|^{2}\right) \\
& =\sigma^{2}\left|u^{n}-u^{n-1}\right|^{2} .
\end{aligned}
$$

(ii) $\mathcal{A}_{a}^{n-1} \cap \mathcal{I}^{n}$ : By construction we have $\lambda^{n}=0, u^{n-1}=a$ and $0 \leq \lambda^{n-1} \leq \sigma(b-a)$ by the definition of $\mathcal{I}^{n}$. Therefore,

$$
d^{n}=\left|\left(\sigma\left(u^{n}-b\right)\right)_{+}\right|^{2}+\left|\left(\sigma\left(a-u^{n}\right)\right)_{+}\right|^{2} .
$$

We distinguish three cases:

( $\alpha) u^{n}<a$. Then

$$
d^{n}=\sigma^{2}\left|a-u^{n}\right|^{2}=\sigma^{2}\left|u^{n-1}-u^{n}\right|^{2} .
$$

( $\beta) a \leq u^{n} \leq b$. Then $d^{n}=0$.

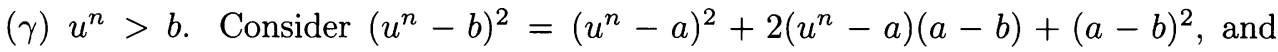
$u^{n}-a>b-a \geq 0$ implying $\left(u^{n}-a\right)(a-b)<(b-a)(a-b)=-(a-b)^{2}$. Hence,

$$
\left(u^{n}-b\right)^{2}<\left(u^{n}-a\right)^{2}-(a-b)^{2},
$$


and consequently

$$
d^{n}=\sigma^{2}\left|\left(u^{n}-b\right)_{+}\right|^{2}<\sigma^{2}\left(\left|u^{n}-u^{n-1}\right|^{2}-|a-b|^{2}\right) .
$$

(iii) $\mathcal{A}_{b}^{n-1} \cap \mathcal{I}^{n}$ : By construction we have $\lambda^{n}=0, u^{n-1}=b$ and $0 \geq \lambda^{n-1} \geq \sigma(a-b)$ by the definition of $\mathcal{I}^{n}$. Therefore,

$$
d^{n}=\left|\left(\sigma\left(u^{n}-b\right)\right)_{+}\right|^{2}+\left|\left(\sigma\left(a-u^{n}\right)\right)_{+}\right|^{2} .
$$

We distinguish three cases:

( $\alpha) u^{n}<a$. Analogously to (ii $\left.\gamma\right)$ we find

$$
\left(a-u^{n}\right)^{2}<\left(b-u^{n}\right)^{2}-(a-b)^{2}
$$

yielding

$$
d^{n}=\sigma^{2}\left|a-u^{n}\right|^{2}<\sigma^{2}\left(\left|u^{n-1}-u^{n}\right|^{2}-|a-b|^{2}\right) .
$$

( $\beta) a \leq u^{n} \leq b$. Then $d^{n}=0$.

$(\gamma) u^{n}>b$. Then

$$
d^{n}=\sigma^{2}\left|u^{n}-b\right|^{2}=\sigma^{2}\left|u^{n}-u^{n-1}\right|^{2} .
$$

(iv) $\mathcal{T}^{n-1}=\mathcal{I}^{n-1} \cap \mathcal{A}^{n}$. We split $\mathcal{A}^{n}$ into its disjoint components $\mathcal{A}_{a}^{n}$ and $\mathcal{A}_{b}^{n}$ and consider therefore the following two cases:

( $\alpha) \mathcal{I}^{n-1} \cap \mathcal{A}_{a}^{n}$. By construction, $\lambda^{n-1}=0, u^{n}=a$, and $u^{n-1}<a$ by definition of $\mathcal{A}_{a}^{n}$. Hence

$$
d^{n}=\left|\left(-\lambda_{-}^{n}\right)_{+}\right|^{2}-\left|\lambda_{-}^{n}\right|^{2}-\left|\left(\sigma\left(a-u^{n-1}\right)\right)_{+}\right|^{2}=-\sigma^{2}\left|u^{n}-u^{n-1}\right|^{2} .
$$

( $\beta) \mathcal{I}^{n-1} \cap \mathcal{A}_{b}^{n}$. By construction, $\lambda^{n-1}=0, u^{n}=b$, and $u^{n-1}>b$ by definition of $\mathcal{A}_{b}^{n}$. Hence

$$
d^{n}=\left|\left(\lambda_{+}^{n}\right)_{+}\right|^{2}-\left|\lambda_{+}^{n}\right|^{2}-\left|\left(\sigma\left(u^{n-1}-b\right)\right)_{+}\right|^{2}=-\sigma^{2}\left|u^{n}-u^{n-1}\right|^{2} .
$$

(v) $\mathcal{A}^{n-1} \cap \mathcal{A}^{n}$. Since the active set of each iteration level can be decomposed into two disjoint sets, the following four cases have to be considered:

( $\alpha) \mathcal{A}_{a}^{n-1} \cap \mathcal{A}_{a}^{n}$. By construction of the iterates, we have $u^{n}=u^{n-1}=a$, and $\lambda^{n-1}<0$ by the definition of $\mathcal{A}_{a}^{n}$. Therefore

$$
d^{n}=\left|\left(-\lambda_{-}^{n}\right)_{+}\right|^{2}-\left|\lambda_{-}^{n}\right|^{2}=0 .
$$

( $\beta) \mathcal{A}_{a}^{n-1} \cap \mathcal{A}_{b}^{n}$. By construction, we have $u^{n-1}=a, u^{n}=b$, and $\lambda^{n-1}>\sigma(b-a)>0$ by definition of $\mathcal{A}_{b}^{n}$. Hence

$$
d^{n}=\left|\left(\lambda_{+}^{n}\right)_{+}\right|^{2}-\left|\lambda_{+}^{n}\right|^{2}=0 .
$$

( $\gamma) \mathcal{A}_{b}^{n-1} \cap \mathcal{A}_{a}^{n}$. This is the reverse situation to (v $\left.\beta\right)$ yielding $d^{n}=0$.

( $\delta) \mathcal{A}_{b}^{n-1} \cap \mathcal{A}_{b}^{n}$. By construction, $u^{n}=u^{n-1}=b$ and $\lambda^{n-1}>0$. This yields

$$
d^{n}=\left|\left(\lambda_{+}^{n}\right)_{+}\right|^{2}-\left|\lambda_{+}^{n}\right|^{2}=0 .
$$

The cases (i), (ii $\alpha$ ), and (iii $\gamma$ ) yield (4.4a). Assertion (4.4b) follows from (ii $\beta$ ), (iii $\beta$ ), and $(\mathrm{v})$, and (4.4c) comes from (ii $\gamma)$ and (iii $\alpha$ ). Finally, case (iv) yields (4.4d).

To estimate the descent in the modified augmented Lagrangian functional, we need another estimate on the set $\mathcal{I}_{*}^{n}$. The next lemma provides the desired result. 
LEMmA 4.4. Let $\left(y^{i}, u^{i}, \lambda^{i}, p^{i}\right), i=n, n-1$ denote two successive iterates of Algorithm AS. Then

$$
d^{n}(x) \leq \sigma^{2}\left|u^{n}(x)-u^{n-1}(x)\right|^{2} \leq \frac{\sigma^{2}}{\alpha^{2}}\left|\tau p^{n}(x)-\tau p^{n-1}(x)\right|^{2} \quad \text { for a.a. } x \in \mathcal{I}_{*}^{n} .
$$

Proof. We again skip the argument $x$ and argue for almost all $x$ like in the previous proof. The basic tool is Eq. (3.2b), which we repeat for convenience:

$$
\alpha u^{n}+\lambda^{n}-\tau p_{\mid \Gamma_{1}}^{n}=\alpha u_{d} \quad \text { on } \Gamma_{1} .
$$

We consider now the components of $\mathcal{I}_{*}^{n}$. In all cases, the first inequality in the assertion of the lemma comes from Lemma 4.3 .

(i) $\mathcal{I}^{n-1} \cap \mathcal{I}^{n}$. From $(3.2 \mathrm{~b})$ and $\lambda^{n}=\lambda^{n-1}=0$ we obtain

$$
u^{n}=\frac{1}{\alpha} \tau p^{n}+u_{d} \quad \text { and } \quad u^{n-1}=\frac{1}{\alpha} \tau p^{n-1}+u_{d}
$$

Therefore,

$$
d^{n} \leq \sigma^{2}\left|u^{n}-u^{n-1}\right|^{2}=\frac{\sigma^{2}}{\alpha^{2}}\left|\tau p^{n}-\tau p^{n-1}\right|^{2} .
$$

(ii) $\mathcal{A}_{a}^{n-1} \cap \mathcal{I}_{-}^{n}$. We have $u^{n}=\frac{1}{\alpha} \tau p^{n}+u_{d}$ by $(3.2 \mathrm{~b})$ with $\lambda^{n}=0$ by construction. Again, from $(3.2 \mathrm{~b})$ we obtain $\tau p^{n-1}-\alpha\left(u^{n-1}-u_{d}\right)=\lambda^{n-1} \geq 0$. This implies $a-u_{d} \leq \frac{1}{\alpha} \tau p^{n-1}$. Therefore,

since

$$
d^{n}=\sigma^{2}\left|a-u^{n}\right|^{2} \leq \frac{\sigma^{2}}{\alpha^{2}}\left|\tau p^{n-1}-\tau p^{n}\right|^{2}
$$

$$
0<u^{n-1}-u^{n}=a-u^{n}=a-\frac{1}{\alpha} \tau p^{n}-u_{d} \leq \frac{1}{\alpha}\left(\tau p^{n-1}-\tau p^{n}\right) .
$$

(iii) $\mathcal{A}_{b}^{n-1} \cap \mathcal{I}_{+}^{n}$. Again, we have $u^{n}=\frac{1}{\alpha} \tau p^{n}+u_{d}$ and $\tau p^{n-1}-\alpha\left(u^{n-1}-u_{d}\right)=\lambda^{n-1} \leq 0$ due to $(3.2 \mathrm{~b})$. A similar argument to case (ii) yields

$$
d^{n}=\sigma^{2}\left|u^{n}-b\right|^{2} \leq \frac{\sigma^{2}}{\alpha^{2}}\left|\tau p^{n}-\tau p^{n-1}\right|^{2},
$$

completing the proof.

The use of Lemma 4.4 relies on the following estimate: Consider (3.2a), which denotes the adjoint equation, yielding

$$
-\Delta \delta_{p}^{n}+c \delta_{p}^{n}=-\delta_{y}^{n} \quad \text { in } \Omega, \quad \frac{\partial \delta_{p}^{n}}{\partial n}=0 \quad \text { on } \Gamma_{1}, \quad \delta_{p}^{n}=0 \quad \text { on } \Gamma_{2},
$$

where $\delta_{p}^{n}=p^{n}-p^{n-1}$ and $\delta_{y}^{n}=y^{n}-y^{n-1}$. This equation is understood in the weak sense, i.e.,

$$
\left(\nabla \delta_{p}^{n}, \nabla v\right)_{\Omega}+\left(c \delta_{p}^{n}, v\right)=\left(-\delta_{y}^{n}, v\right) \text { for all } v \in V .
$$

The $V$-ellipticity of the bilinear form $a(w, v)=(\nabla w, \nabla v)_{\Omega}+(c w, v)_{\Omega}$ and $\bar{c} \geq c \geq 0$ imply

$$
\left|\delta_{p}^{n}\right|_{H^{1}(\Omega)} \leq \tilde{c}\left|\delta_{y}^{n}\right|_{\Omega},
$$

with some constant $\tilde{c}>0$. Let $|\tau|$ denote the operator norm of the trace operator $\tau: H^{1}(\Omega) \rightarrow L^{2}(\Gamma)$. Then

$$
\left|\tau p^{n}-\tau p^{n-1}\right|_{\Gamma_{1}} \leq|\tau|\left|\delta_{p}^{n}\right|_{H^{1}(\Omega)} \leq C\left|\delta_{y}^{n}\right|_{\Omega},
$$

with $C:=|\tau| \tilde{c}$. 
Now we are prepared to prove the main result of this section. The following theorem gives conditions on the parameters $\alpha$ and $\sigma$ for the modified augmented Lagrangian to be non-ascending.

ThEOREM 4.5. Suppose that $\mathcal{A}^{n} \neq \mathcal{A}^{n-1}$ (in the sense of (3.1)). If

$$
\rho+\alpha \leq \sigma \leq \alpha+\frac{\alpha^{2}}{C^{2}}-\frac{\alpha^{2}}{\rho} \quad \text { and } \quad(\sigma-\alpha)\left|u^{n-1}-u^{n}\right|_{\mathcal{P}^{n}}^{2}-\sigma|a-b|_{\mathcal{P}^{n}}^{2} \leq 0
$$

for some $\rho>0$, then

$$
L\left(y^{n}, u^{n}, \lambda^{n}\right)-L\left(y^{n-1}, u^{n-1}, \lambda^{n-1}\right) \leq 0 .
$$

Proof. We first relax the bound on $d^{n}$ in two specific situations. In (i) and (ii) below, we skip the argument $x$ for convenience.

(i) $\mathcal{A}_{a}^{n-1} \cap \mathcal{A}_{b}^{n}$. We have $u^{n-1}=a$ and $u^{n}=b$ by construction and, as a consequence of the definition of $\mathcal{A}_{b}^{n}$, we obtain $\lambda^{n-1}>\sigma(b-a)>0$. Therefore,

$$
\begin{aligned}
-\sigma^{2}\left|u^{n-1}-u^{n}\right|^{2}+2 \sigma\left(u^{n-1}-u^{n}\right)\left(-\lambda^{n-1}\right) & =-\sigma^{2}|a-b|^{2}+2 \sigma(b-a)\left(\lambda^{n-1}\right) \\
& >-\sigma^{2}|a-b|^{2}+2 \sigma(b-a)(\sigma(b-a)) \\
& =\sigma^{2}|a-b|^{2}>d^{n} .
\end{aligned}
$$

(ii) $\mathcal{A}_{b}^{n-1} \cap \mathcal{A}_{a}^{n}$. In this case we have $u^{n-1}=b, u^{n}=a$, and $\lambda^{n-1}<\sigma(a-b)<0$. An analogous computation to (i) results again in

$$
-\sigma^{2}\left|u^{n-1}-u^{n}\right|^{2}+2 \sigma\left(u^{n-1}-u^{n}\right)\left(-\lambda^{n-1}\right)>d^{n}
$$

Since $\lambda^{n-1}=0$ on $\mathcal{T}^{n-1}$, the estimate (4.8) (with ">" replaced by " $\geq$ ") trivially holds on $\mathcal{T}^{n-1}$ due to $(4.4 \mathrm{~d})$. Moreover, observe that $\left(\mathcal{A}_{a}^{n-1} \cap \mathcal{A}_{b}^{n}\right) \cup\left(\mathcal{A}_{b}^{n-1} \cap \mathcal{A}_{a}^{n}\right) \cup \mathcal{T}^{n-1}=\mathcal{A}_{*}^{n}$ (for its definition, see Corollary 4.2).

Next we compute an upper bound to the inner product $\left(u^{n-1}-u^{n}, \lambda^{n}-\lambda^{n-1}\right)_{\mathcal{A}_{*}^{n}}$ by

$$
\begin{aligned}
\left(u^{n-1}-u^{n}, \lambda^{n}-\lambda^{n-1}\right)_{\mathcal{A}_{*}^{n}} & =\left(u^{n-1}-u^{n}, \tau p^{n}-\tau p^{n-1}\right)_{\mathcal{A}_{*}^{n}}+\alpha\left|u^{n-1}-u^{n}\right|_{\mathcal{A}_{*}^{n}}^{2} \\
& \leq\left|\tau p^{n}-\tau p^{n-1}\right|_{\Gamma_{1}}\left|u^{n-1}-u^{n}\right|_{\mathcal{A}_{*}^{n}}^{n}+\alpha\left|u^{n-1}-u^{n}\right|_{\mathcal{A}_{*}^{n}}^{2} \\
& \leq C\left|\delta_{y}^{n}\right|_{\Omega}\left|u^{n-1}-u^{n}\right|_{\mathcal{A}_{*}^{n}}+\alpha\left|u^{n-1}-u^{n}\right|_{\mathcal{A}_{*}^{n}}^{2}
\end{aligned}
$$

where we used (3.2b) and then (4.6). 
Now let us estimate the difference of the modified augmented Lagrangian functional for two successive iterates:

$$
\begin{aligned}
& L\left(y^{n}, u^{n}, \lambda^{n}\right)-L\left(y^{n-1}, u^{n-1}, \lambda^{n-1}\right) \\
& \stackrel{(4.2)}{=}-\frac{1}{2}\left|\delta_{y}^{n}\right|_{\Omega}^{2}-\frac{\alpha}{2}\left|\delta_{u}^{n}\right|_{\Gamma_{1}}^{2}+\left(u^{n-1}-u^{n}, \lambda^{n}\right)_{\mathcal{A}_{*}^{n}}+\frac{1}{2 \sigma} \int_{\Gamma_{1}} d^{n} d s \\
& \stackrel{(4.4)}{\leq}-\frac{1}{2}\left|\delta_{y}^{n}\right|_{\Omega}^{2}-\frac{\alpha}{2}\left|\delta_{u}^{n}\right|_{\Gamma_{1}}^{2}+\left(u^{n-1}-u^{n}, \lambda^{n}\right)_{\mathcal{A}_{*}^{n}}+\frac{\sigma}{2}\left|\delta_{u}^{n}\right|_{\mathcal{I}_{*}^{n}}^{2}-\frac{\sigma}{2}\left|\delta_{u}^{n}\right|_{\mathcal{T}^{n-1}}^{2} \\
& \quad+\left(u^{n-1}-u^{n},-\lambda^{n-1}\right)_{\mathcal{T}^{n-1}}+\frac{\sigma}{2}\left|\delta_{u}^{n}\right|_{\mathcal{P}^{n}}^{2}-\frac{\sigma}{2}|a-b|_{\mathcal{P}^{n}}^{2}-\frac{\sigma}{2}\left|\delta_{u}^{n}\right|_{\mathcal{A}_{a}^{n-1} \cap \mathcal{A}_{b}^{n}}^{2} \\
& \quad+\left(u^{n-1}-u^{n},-\lambda^{n-1}\right)_{\mathcal{A}_{a}^{n-1} \cap \mathcal{A}_{b}^{n}}-\frac{\sigma}{2}\left|\delta_{u}^{n}\right|_{\mathcal{A}_{b}^{n-1} \cap \mathcal{A}_{a}^{n}}^{2}+\left(u^{n-1}-u^{n},-\lambda^{n-1}\right)_{\mathcal{A}_{b}^{n-1} \cap \mathcal{A}_{a}^{n}} \\
&=-\frac{1}{2}\left|\delta_{y}^{n}\right|_{\Omega}^{2}-\frac{\alpha}{2}\left|\delta_{u}^{n}\right|_{\Gamma_{1}}^{2}+\left(u^{n-1}-u^{n}, \lambda^{n}-\lambda^{n-1}\right)_{\mathcal{A}_{*}^{n}}+\frac{\sigma}{2}\left|\delta_{u}^{n}\right|_{\mathcal{I}_{*}^{n}}^{2}-\frac{\sigma}{2}\left|\delta_{u}^{n}\right|_{\mathcal{A}_{*}^{n}}^{2} \\
& \quad+\frac{\sigma}{2}\left|\delta_{u}^{n}\right|_{\mathcal{P}^{n}}^{2}-\frac{\sigma}{2}|a-b|_{\mathcal{P}^{n}}^{2} \\
& \leq\left(C^{2} \frac{\sigma-\alpha}{2 \alpha^{2}}-\frac{1}{2}\right)\left|\delta_{y}^{n}\right|_{\Omega}^{2}+C\left|\delta_{y}^{n}\right|_{\Omega}\left|\delta_{u}^{n}\right|_{\mathcal{A}_{*}^{n}}+\frac{1}{2}(\alpha-\sigma)\left|\delta_{u}^{n}\right|_{\mathcal{A}_{*}^{n}}^{2} \\
&+\frac{1}{2}(\sigma-\alpha)\left|\delta_{u}^{n}\right|_{\mathcal{P}^{n}}^{2}-\frac{\sigma}{2}|a-b|_{\mathcal{P}^{n}}^{2}-\frac{\alpha}{2}\left|\delta_{u}^{n}\right|_{\Gamma_{1} \backslash\left(\mathcal{I}_{*}^{n} \cup \mathcal{A}_{*}^{n} \cup \mathcal{P}^{n}\right)}^{2}=: \Psi^{n} .
\end{aligned}
$$

For the last inequality above, we used (4.5), (4.6), (4.9) and $\sigma \geq \alpha$. We continue by making use of $s t \leq \frac{1}{2}\left(s^{2} \kappa^{-1}+\kappa t^{2}\right)$ for every $\kappa>0$ and obtain

$$
\begin{aligned}
\Psi^{n} \leq & \frac{1}{2}\left(C^{2} \frac{\sigma-\alpha}{\alpha^{2}}-1\right)\left|\delta_{y}^{n}\right|_{\Omega}^{2}+\frac{C}{2 \kappa}\left|\delta_{y}^{n}\right|_{\Omega}^{2}+\frac{C \kappa}{2}\left|\delta_{u}^{n}\right|_{\mathcal{A}_{*}^{n}}^{2}+\frac{1}{2}(\alpha-\sigma)\left|\delta_{u}^{n}\right|_{\mathcal{A}_{*}^{n}}^{2} \\
& +\frac{1}{2}(\sigma-\alpha)\left|\delta_{u}^{n}\right|_{\mathcal{P}^{n}}^{2}-\frac{\sigma}{2}|a-b|_{\mathcal{P}^{n}}^{2} \\
= & \frac{1}{2}\left(\frac{C}{\kappa}+C^{2} \frac{\sigma-\alpha}{\alpha^{2}}-1\right)\left|\delta_{y}^{n}\right|_{\Omega}^{2}+\frac{1}{2}(C \kappa+\alpha-\sigma)\left|\delta_{u}^{n}\right|_{\mathcal{A}_{*}^{n}}^{2}+\frac{1}{2}(\sigma-\alpha)\left|\delta_{u}^{n}\right|_{\mathcal{P}^{n}}^{2}-\frac{\sigma}{2}|a-b|_{\mathcal{P}^{n}}^{2} .
\end{aligned}
$$

If we set $\rho=C \kappa$, then $L\left(y^{n}, u^{n}, \lambda^{n}\right)-L\left(y^{n-1}, u^{n-1}, \lambda^{n-1}\right) \leq 0$ provided that

$$
\left(\frac{C^{2}}{\rho}+C^{2} \frac{\sigma-\alpha}{\alpha^{2}}-1\right) \leq 0 \text { and } \rho+\alpha-\sigma \leq 0
$$

and, whenever $\mathcal{P}^{n}$ is nonempty,

$$
(\sigma-\alpha)\left|\delta_{u}^{n}\right|_{\mathcal{P}^{n}}^{2}-\sigma|a-b|_{\mathcal{P}^{n}}^{2} \leq 0 .
$$

This proves the assertion.

As an important consequence of Theorem 4.5 we obtain

Corollary 4.6. Suppose that $\mathcal{A}^{n} \neq \mathcal{A}^{n-1}$ (in the sense of (3.1)). If

$$
\rho+\alpha \leq \sigma<\alpha+\frac{\alpha^{2}}{C^{2}}-\frac{\alpha^{2}}{\rho} \quad \text { and } \quad(\sigma-\alpha)\left|u^{n-1}-u^{n}\right|_{\mathcal{P}^{n}}^{2}-\sigma|a-b|_{\mathcal{P}^{n}}^{2} \leq 0
$$

for some $\rho>0$, then

$$
L\left(y^{n}, u^{n}, \lambda^{n}\right)-L\left(y^{n-1}, u^{n-1}, \lambda^{n-1}\right)<0 .
$$


If $\alpha$ and $\sigma$ satisfy the conditions of Corollary 4.6, then the modified augmented Lagrangian functional is strictly decreasing. This fact prevents Algorithm AS from chattering, i.e., the algorithm will never compute the same active and inactive sets twice.

Next, we consider the uniform boundedness of the iterates of Algorithm AS. For this purpose observe that the iterates are obtained as solutions to the auxiliary problem

$$
\left\{\begin{array}{l}
\text { minimize } J(y, u) \\
\text { such that }-\Delta y+c y=g \text { in } \Omega, \quad \frac{\partial y}{\partial n}=u \text { on } \Gamma_{1}, \quad y=0 \text { on } \Gamma_{2}, \\
\text { and } u_{\mid \mathcal{A}_{a}^{n}}=a_{\mid \mathcal{A}_{a}^{n}}, \quad u_{\mid \mathcal{A}_{b}^{n}}=b_{\mid \mathcal{A}_{b}^{n}}
\end{array}\right.
$$

with $\lambda_{\mid \mathcal{I}^{n}}=0$. The following lemma exploits this fact.

Lemma 4.7. The iterates $\left(y^{n}, u^{n}, p^{n}, \lambda^{n}\right), n \in \mathbb{N}$, of Algorithm AS are uniformly bounded, i.e., there exists a constant $\beta \in \mathbb{R}_{+}$such that

$$
\max \left\{\left|y^{n}\right|_{H^{1}(\Omega)},\left|u^{n}\right|_{\Gamma_{1}},\left|p^{n}\right|_{H^{1}(\Omega)},\left|\lambda^{n}\right|_{\Gamma_{1}}\right\} \leq \beta \quad \text { for all } n .
$$

Proof. First we construct a feasible pair $\left(\tilde{y}^{n}, \tilde{u}^{n}\right)$ for the auxiliary problem (4.11) at each iteration level. First this aim put $\tilde{u}_{\mid \mathcal{A}_{a}^{n}}^{n}=a_{\mid \mathcal{A}_{a}^{n}}, \tilde{u}_{\mid \mathcal{A}_{b}^{n}}^{n}=b_{\mid \mathcal{A}_{b}^{n}}$, and $\tilde{u}_{\mid \mathcal{I}^{n} \cup \Gamma_{2}}^{n}=0$. Moreover, let $\tilde{y}^{n}$ be the unique solution of

$$
-\Delta y+c y=g \quad \text { in } \Omega, \quad \frac{\partial y}{\partial n}=\tilde{u}^{n} \quad \text { on } \Gamma_{1}, \quad y=0 \quad \text { on } \Gamma_{2} .
$$

Since $a, b \in L^{\infty}\left(\Gamma_{1}\right)$, by assumption there exist constants $\beta_{a}, \beta_{b} \in \mathbb{R}_{+}$such that

$$
\left|\tilde{u}^{n}\right|_{\Gamma_{1}} \leq \beta_{a}+\beta_{b} \text { for all } n .
$$

To prove the uniform boundedness of $\tilde{y}^{n}, n \in \mathbb{N}$, we choose $\tilde{y}_{o}^{n} \in H^{1}(\Delta, \Omega$ ) (for its definition, see Appendix A) such that

$$
\frac{\partial \tilde{y}_{o}^{n}}{\partial n}=\tilde{u}^{n} \quad \text { on } \Gamma_{1}
$$

Next we define $\tilde{y}_{s}^{n}$ as the unique solution to

$$
-\Delta y+c y=g+\Delta \tilde{y}_{o}^{n}-c \tilde{y}_{o}^{n} \quad \text { in } \Omega, \quad \frac{\partial y}{\partial n}=0 \quad \text { on } \Gamma_{1}, \quad y=0 \quad \text { on } \Gamma_{2} .
$$

Let $|\Delta|$ denote the operator norm of $\Delta: H^{1}(\Delta, \Omega) \rightarrow L^{2}(\Omega)$. Then we obtain the bound

$$
\left|\tilde{y}_{s}^{n}\right|_{H^{1}(\Omega)} \leq c_{1}\left(|g|_{\Omega}+|\Delta|\left|\tilde{y}_{o}^{n}\right|_{H^{1}(\Delta, \Omega)}+\tilde{c}\left|\tilde{y}_{o}^{n}\right|_{\Omega}\right)
$$

where $c_{1}>0$ denotes a suitable constant. Let $\omega: H^{-\frac{1}{2}}(\Gamma) \rightarrow H^{1}(\Delta, \Omega)$ define the continuous lifting operator with its operator norm denoted by $|\omega|$. Then

$$
\left|\tilde{y}_{o}^{n}\right|_{\Omega} \leq\left|\tilde{y}_{o}^{n}\right|_{H^{1}(\Delta, \Omega)} \leq c_{2}|\omega|\left|\tilde{u}^{n}\right|_{\Gamma_{1}} \leq c_{2}\left(\beta_{a}+\beta_{b}\right)|\omega|
$$

for all $n \in \mathbb{N}$, where $c_{2}>0$ is a suitable constant. Now observe that $\tilde{y}^{n}=\tilde{y}_{s}^{n}+\tilde{y}_{o}^{n}$ and that this fact, together with (4.12) and (4.13), yields the uniform bound to $\tilde{y}^{n}$ in $H^{1}(\Omega)$.

Since $\left(y^{n}, u^{n}\right)$ is the unique solution to the auxiliary problem (4.11) we have

$$
0 \leq J\left(y^{n}, u^{n}\right) \leq J\left(\tilde{y}^{n}, \tilde{u}^{n}\right) \leq \beta_{J},
$$

where the nonnegativity of $J$ follows from its definition, and the existence of the constant $\beta_{J}>0$ is due to the uniform boundedness of $\left(\tilde{y}^{n}, \tilde{u}^{n}\right)$. This proves the uniform boundedness of $y^{n}$ and $y^{n}$ in $L^{2}(\Omega)$ and $L^{2}\left(\Gamma_{1}\right)$, respectively. 
A similar procedure as in the proof of the uniform boundedness of $\tilde{y}^{n}$ in $H^{1}(\Omega)$ (now $u^{n}$ taking the role of $\tilde{u}^{n}$ ) yields the uniform boundedness of $y^{n}$ in $H^{1}(\Omega)$.

Next consider the adjoint equation at each iteration level, i.e.,

$$
-\Delta p+c p=z_{d}-y^{n} \quad \text { in } \Omega, \quad \frac{\partial p}{\partial n}=0 \quad \text { on } \Gamma_{1}, \quad p=0 \quad \text { on } \Gamma_{2}
$$

with $p^{n}$ its unique solution in the weak sense. Then

$$
\left|p^{n}\right|_{H_{1}(\Omega)} \leq \tilde{c}\left|z_{d}-y^{n}\right|_{\Omega},
$$

proving the uniform boundedness of $p^{n}$ in $H^{1}(\Omega)$.

Finally, the uniform boundedness of $\lambda^{n}$ in $L^{2}\left(\Gamma_{1}\right)$ follows from

$$
\left|\lambda^{n}\right|_{\Gamma_{1}} \leq|\tau|\left|p^{n}\right|_{H^{1}(\Omega)}+\alpha\left(\left|u_{d}\right|_{\Gamma_{1}}+\left|u^{n}\right|_{\Gamma_{1}}\right) \text { for all } n
$$

where $|\tau|$ denotes the operator norm of the zero-order trace operator $\tau: H^{1}(\Omega) \rightarrow$ $H^{1 / 2}(\Gamma)$.

Note that due to $z_{d}, g \in L^{2}(\Omega)$ and $y^{n} \in H^{1}(\Omega)$, for all $n \in \mathbb{N}$, in (4.14) we even obtain

$$
\begin{aligned}
& p^{n} \in \mathcal{W} \quad \text { and }\left|p^{n}\right|_{H^{2}(\Omega)} \leq c_{3}\left(\left|z_{d}-y^{n}\right|_{\Omega}+\left|p^{n}\right|_{H^{1}(\Omega)}\right) \leq c_{3}\left(\left|z_{d}\right|_{\Omega}+2 \beta\right), \\
& y^{n} \in \mathcal{W} \text { and }\left|y^{n}\right|_{H^{2}(\Omega)} \leq c_{4}\left(|g|_{\Omega}+\left|y^{n}\right|_{H^{1}(\Omega)}\right) \leq c_{4}\left(|g|_{\Omega}+\beta\right)
\end{aligned}
$$

with $\mathcal{W}=H^{2}(\Omega) \cap V$ and suitable $c_{3}, c_{4} \in \mathbb{R}_{+}$, under suitable assumptions on the boundary from regularity results for elliptic equations; see [24], [10].

Next, we turn towards the convergence of the iterates $\left(y^{n}, u^{n}, p^{n}, \lambda^{n}\right)$. We assume that $\mathcal{A}^{n+1} \neq \mathcal{A}^{n}$ for all $n \in \mathbb{N}$, because otherwise Lemma 3.1 proves the actual iterate to be the unique optimal solution of (1.1). We prove first an auxiliary result based on Corollary 4.6 and Theorem 4.5.

Lemma 4.8. Suppose that $\mathcal{A}^{n} \neq \mathcal{A}^{n-1}$ for all $n \in \mathbb{N}$, and that there exists $\tilde{\epsilon}>0$ such that for all $n \in \mathbb{N}$,

and

$$
\rho+\alpha<\sigma<\alpha+\frac{\alpha^{2}}{C^{2}}-\frac{\alpha^{2}}{\rho}
$$

$$
(\sigma-\alpha)\left|\delta_{u}^{n}\right|_{\mathcal{P}^{n}}^{2}-\sigma|a-b|_{\mathcal{P}^{n}}^{2} \leq-\tilde{\epsilon}\left|\delta_{u}^{n}\right|_{\mathcal{P}^{n}}^{2}<0
$$

whenever $\mathcal{P}^{n} \neq \varnothing$. Then

(i) $\lim _{n \rightarrow \infty}\left|\delta_{y}^{n}\right|_{\Omega}=0$,

(ii) $\lim _{n \rightarrow \infty}\left|\tau \delta_{p}^{n}\right|_{\Gamma_{1}}=0$,

(iii) $\lim _{n \rightarrow \infty}\left|\delta_{u}^{n}\right|_{\Gamma_{1}}=0$.

Proof. From Corollary 4.6 we obtain that $\left\{L\left(y^{n}, u^{n}, \lambda^{n}\right)\right\}_{n=0}^{\infty}$ is strictly decreasing. Since $L(y, u, \lambda)$ is uniformly bounded from below, there exists $L^{*}$ such that $\left(L\left(y^{n}, u^{n}, \lambda^{n}\right)\right.$ $\downarrow L^{*}$. From the proof of Theorem 4.5 we infer that

$$
L\left(y^{n}, u^{n}, \lambda^{n}\right)-L\left(y^{n-1}, u^{n-1}, \lambda^{n-1}\right) \leq \frac{1}{2}\left(\frac{C^{2}}{\rho}+C^{2} \frac{\sigma-\alpha}{\alpha^{2}}-1\right)\left|\delta_{y}^{n}\right|_{\Omega}^{2}<0 .
$$

Since the left-hand side vanishes as $n \rightarrow \infty$, assertion (i) is proved.

For the proof of (ii), we consider (4.6). Then the result immediately follows from (i). 
Note that $\left|\delta_{u}^{n}\right|_{\mathcal{A}^{n} \backslash \mathcal{A}_{*}^{n}}=0$ for all $n \in \mathbb{N}$. From the choice of $\sigma$, Lemma 4.4 and (ii) we obtain

$$
\lim _{n \rightarrow \infty}\left|\delta_{u}^{n}\right|_{\mathcal{A}_{*}^{n} \cup \mathcal{I}_{*}^{n} \cup \mathcal{P}^{n}}=0
$$

Finally, from the definition of $\Psi^{n}$ in the proof of Theorem 4.5 we obtain $\lim _{n \rightarrow \infty}\left|\delta_{u}^{n}\right| \mathcal{F}_{a}^{n} \cup \mathcal{F}_{b}^{n}$ $=0$. Combining all results for $\delta_{u}^{n}$ we obtain (iii).

The convergence result for the iterates of Algorithm AS is given in the next theorem.

Theorem 4.9. Suppose that $\operatorname{essinf}\left\{b(x)-a(x) \mid x \in \Gamma_{1}\right\} \geq \underline{\epsilon}>0$ and that the assumptions of Lemma 4.8 are satisfied. Then $\left\{\left(y^{n}, p^{n}\right)\right\}_{n=0}^{\infty}$ converges to $\left(y^{*}, p^{*}\right)$ strongly in $V$, and $\left\{\left(u^{n}, \lambda^{n}\right)\right\}_{n=0}^{\infty}$ converges to $\left(u^{*}, \lambda^{*}\right)$ weakly in $L^{2}\left(\Gamma_{1}\right)$.

Proof. The proof is based on Lemma 4.8, i.e., the fact that the sequences of increments $\left\{\delta_{y}^{n}\right\},\left\{\delta_{u}^{n}\right\}$, and $\left\{\tau \delta_{p}^{n}\right\}$ converge to zero in the respective norms without assuming that the sequences $\left\{y^{n}\right\},\left\{u^{n}\right\}$, and $\left\{p^{n}\right\}$ converge.

By Lemma 4.7, (4.15a) and (4.15b), the sequence $\left\{\left(y^{n}, u^{n}, p^{n}, \lambda^{n}\right)\right\}_{n=0}^{\infty}$ is uniformly bounded in $\mathcal{W} \times L^{2}\left(\Gamma_{1}\right) \times \mathcal{W} \times L^{2}\left(\Gamma_{1}\right)$. Hence there exist a subsequence $\{n(k)\}_{k=0}^{\infty}$ and $(\bar{y}, \bar{u}, \bar{p}, \bar{\lambda})$ such that $\left\{\left(y^{n(k)}, p^{n(k)}\right)\right\}_{k=0}^{\infty}$ converges to $(\bar{y}, \bar{p})$ strongly in $V$ due to the compact embedding of $H^{2}(\Omega)$ in $H^{1}(\Omega)$, and such that $\left\{\left(u^{n(k)}, \lambda^{n(k)}\right)\right\}_{k=0}^{\infty}$ converges to $(\bar{u}, \bar{\lambda})$ weakly in $L^{2}\left(\Gamma_{1}\right)$. By construction of the iteration sequence we have

$$
\begin{array}{rlrl}
-\Delta \bar{p}+c \bar{p}+\bar{y} & =z_{d} \text { in } \Omega, & \frac{\partial \bar{p}}{\partial n}=0 \text { on } \Gamma_{1}, & \bar{p}=0 \text { on } \Gamma_{2}, \\
\alpha \bar{u}+\bar{\lambda}-\tau \bar{p}_{\mid \Gamma_{1}} & =\alpha u_{d} \text { on } \Gamma_{1}, \\
-\Delta \bar{y}+c \bar{y} & =g \text { in } \Omega, \quad \frac{\partial \bar{y}}{\partial n}=\bar{u} \text { on } \Gamma_{1}, \quad \bar{y}=0 \text { on } \Gamma_{2} .
\end{array}
$$

Thus, primal and dual feasibility remains to be considered. We start by proving $\bar{u} \in U_{a d}$, i.e., primal feasibility. For this purpose choose an arbitrary $\epsilon>0$, and let

$$
U^{n}=\left\{x \in \Omega \mid u^{n} \leq a-\epsilon \text { or } u^{n} \geq b+\epsilon\right\} .
$$

Assume that meas $\left(U^{n(k(l))}\right) \geq \delta^{2}>0$ along a subsequence $\{n(k(l))\}_{l=0}^{\infty}$ of $\{n(k)\}_{k=0}^{\infty}$. Without loss of generality, we assume $n(k(l))=n(k)$. Then due to $U^{n} \subset\left(\mathcal{I}^{n} \cap \mathcal{A}^{n+1}\right)$, by the definition of $\mathcal{I}^{n}$ and $\mathcal{A}^{n+1}$, we obtain

$$
0<\epsilon \delta \leq \epsilon \sqrt{\operatorname{meas}\left(U^{n(k)}\right)} \leq\left|u^{n(k)+1}-u^{n(k)}\right|_{U^{n}(k)} \leq\left|\delta_{u}^{n(k)+1}\right|_{\Gamma_{1}} \quad \text { for all } k \in \mathbb{N} .
$$

Since $\left|\delta_{u}^{n}\right|_{\Gamma_{1}} \rightarrow 0$ due to Lemma 4.8, we also have $\left|\delta_{n}^{n(k)+1}\right|_{\Gamma_{1}} \rightarrow 0$. This contradicts (4.16). Thus, $\bar{u} \in U_{\text {ad }}$, which establishes primal feasibility.

Finally, we turn towards feasibility of $\bar{\lambda}$. Let $\overline{\mathcal{A}}_{a}=\{x \in \Omega \mid \bar{u}=a\}$ and $\Lambda_{a}^{n}=\{x \in$ $\left.\overline{\mathcal{A}}_{a} \mid \lambda^{n} \geq \epsilon\right\}$ for arbitrarily chosen $\epsilon>0$. Note that $\Lambda_{a}^{n} \subset \mathcal{A}^{n}$ and $\Lambda_{a}^{n} \cap \mathcal{A}_{a}^{n+1}=\varnothing$. Assume that meas $\left(\Lambda_{a}^{n(k(l))} \cap \mathcal{I}^{n(k(l))+1}\right) \geq \delta^{2}>0$ along a subsequence $\{n(k(l))\}_{l=0}^{\infty}$ of $\{n(k)\}_{k=0}^{\infty}$. Again, without loss of generality, we assume $n(k(l))=n(k)$. Then

$$
0<\epsilon \delta \leq\left|\lambda^{n(k)+1}-\lambda^{n(k)}\right|_{\Lambda_{a}^{n(k)} \cap \mathcal{I}^{n(k)+1}} \leq \alpha\left|\delta_{u}^{n(k)+1}\right|_{\Gamma_{1}}+\left|\tau \delta_{p}^{n(k)+1}\right|_{\Gamma_{1}},
$$

where we also used (3.2b). Since $\lim _{n \rightarrow \infty}\left(\alpha\left|\delta_{u}^{n}\right|_{\Gamma_{1}}+\left|\tau \delta_{p}^{n}\right|_{\Gamma_{1}}\right)=0$ by Lemma 4.8, we obtain a contradiction to our assumption on $\left\{\operatorname{meas}\left(\Lambda_{a}^{n(k)} \cap \mathcal{I}^{n(k)+1}\right)\right\}_{k=0}^{\infty}$. Next (again by 
using $n(k(l))=n(k))$ assume that meas $\left(\Lambda_{a}^{n(k)} \cap \mathcal{A}_{a}^{n(k)} \cap \mathcal{A}_{b}^{n(k)+1}\right) \geq \delta^{2}>0$ for all $k \in \mathbb{N}$. Then

$$
0<\delta \underline{\epsilon} \leq|a-b|_{\Lambda_{a}^{n(k)} \cap \mathcal{A}_{a}^{n(k)} \cap \mathcal{A}_{b}^{n(k)+1}} \leq\left|\delta_{u}^{n(k)+1}\right|_{\Gamma_{1}},
$$

which, due to $\lim _{n \rightarrow \infty}\left|\delta_{u}^{n}\right|=0$, yields a contradiction. Finally, assume that meas $\left(\Lambda_{a}^{n(k)} \cap\right.$ $\left.\mathcal{A}_{b}^{n(k)} \cap \mathcal{A}_{b}^{n(k)+1}\right) \geq \delta^{2}>0$ for all $k \in \mathbb{N}$. Then $u^{n(k)}=b$ on $\left(\Lambda_{a}^{n(k)} \cap \mathcal{A}_{b}^{n(k)} \cap \mathcal{A}_{b}^{n(k)+1}\right)$ for all $k \in \mathbb{N}$. Since $\left\{u^{n(k)}\right\}_{k=0}^{\infty}$ converges weakly in $L^{2}\left(\Gamma_{1}\right)$ to $\bar{u}$ with $\bar{u}=a$ on $\overline{\mathcal{A}}_{a}$, a contradiction is obtained. Combining all results for $\overline{\mathcal{A}}_{a}$, we infer $\bar{\lambda}_{\mid \overline{\mathcal{A}}_{a}} \leq 0$.

Similar arguments as for $\overline{\mathcal{A}}_{a}$ yield $\bar{\lambda}_{\mid \overline{\mathcal{A}}_{b}} \geq 0$ with $\overline{\mathcal{A}}_{b}=\{x \in \Omega \mid \bar{u}=b\}$.

Finally, consider $\overline{\mathcal{I}}_{\epsilon}=\{x \in \Omega \mid a+\epsilon \leq \bar{u} \leq b-\epsilon\}$ and $\Lambda_{0}^{n}=\left\{x \in \overline{\mathcal{I}}_{\epsilon}|| \lambda^{n}(x) \mid \geq \epsilon\right.$ a.e. $\}$ for arbitrarily fixed $\epsilon>0$. Note that $\overline{\mathcal{I}}_{\epsilon} \subset \overline{\mathcal{I}}:=\{x \in \Omega \mid a<\bar{u}<b\}$, and $\Lambda_{0}^{n} \subset \mathcal{A}^{n}$. Define

$$
\Lambda_{0, a, b}^{n}=\left(\Lambda_{0}^{n} \cap \mathcal{A}_{a}^{n} \cap \mathcal{A}_{b}^{n+1}\right) \cup\left(\Lambda_{0}^{n} \cap \mathcal{A}_{b}^{n} \cap \mathcal{A}_{a}^{n+1}\right)
$$

and assume that meas $\left(\Lambda_{0, a, b}^{n(k(l))}\right) \geq \delta^{2}>0$ along a subsequence $\{n(k(l))\}_{l=0}^{\infty}$ of $\{n(k)\}_{k=0}^{\infty}$. Without loss of generality, we again assume $n(k(l))=n(k)$. Then

$$
0<\delta \underline{\epsilon} \leq|a-b|_{\Lambda_{0, a, b}^{n(k)}} \leq\left|\delta_{u}^{n(k)+1}\right|_{\Gamma_{1}},
$$

which, due to $\lim _{n \rightarrow \infty}\left|\delta_{u}^{n}\right|=0$, yields a contradiction. Now, with $n(k(l))=n(k)$ assume that meas $\left(\Lambda_{0}^{n(k)} \cap \mathcal{A}_{a}^{n(k)} \cap \mathcal{A}_{a}^{n(k)+1}\right) \geq \delta^{2}>0$ for all $k \in \mathbb{N}$. Then $u^{n(k)}=a$ on $\left(\Lambda_{0}^{n(k)} \cap\right.$ $\left.\mathcal{A}_{a}^{n(k)} \cap \mathcal{A}_{a}^{n(k)+1}\right)$ for all $k \in \mathbb{N}$. But this contradicts $\bar{u} \geq a+\epsilon$ on $\overline{\mathcal{I}}_{\epsilon}$. Analogous arguments apply to $\left(\Lambda_{0}^{n(k)} \cap \mathcal{A}_{b}^{n(k)} \cap \mathcal{A}_{b}^{n(k)+1}\right)$. Thus, when combining all preceding results, we have $\bar{\lambda}_{\mid \overline{\mathcal{I}}}=0$.

In conclusion, $(\bar{y}, \bar{u}, \bar{p}, \bar{\lambda})$ satisfies the first-order system of Theorem 2.3. The assertion then follows from the uniqueness of the optimal solution $\left(y^{*}, u^{*}, p^{*}, \lambda^{*}\right)$ of (1.1).

In the remainder of this section, we check that for given $\alpha$, the interval of $\sigma$-values satisfying (4.10) is nonempty. Let us first closely analyze the condition

$$
(\sigma-\alpha)\left|u^{n-1}-u^{n}\right|_{\mathcal{P}^{n}}^{2}-\sigma|a-b|_{\mathcal{P}^{n}}^{2} \leq 0,
$$

which clearly depends on the iteration sequence. We investigate the components of $\mathcal{P}^{n}$, i.e., $\mathcal{P}_{a}^{n}$ and $\mathcal{P}_{b}^{n}$. First we concentrate on $\mathcal{P}_{a}^{n}$. Since $\mathcal{P}_{a}^{n}$ contains those $x$ for which $u^{n}(x)>b(x)$ a.e., we obtain

$$
\left|u^{n}-u^{n-1}\right|_{\mathcal{P}_{a}^{n}}>|b-a|_{\mathcal{P}_{a}^{n}},
$$

where we additionally used $u_{\mid \mathcal{P}_{a}^{n}}^{n-1}=a_{\mid \mathcal{P}_{a}^{n}}$. Hence (4.17) restricted to $\mathcal{P}_{a}^{n}$ is equivalent to

$$
\sigma \xi_{a}^{n} \leq \alpha \quad \text { with } \xi_{a}^{n} \in(0,1)
$$

where $\xi_{a}^{n}$ is defined as

$$
\xi_{a}^{n}=1-\frac{|b-a|_{\mathcal{P}_{a}^{n}}^{2}}{\left|u^{n}-u^{n-1}\right|_{\mathcal{P}_{a}^{n}}^{2}}=1-\frac{|b-a|_{\mathcal{P}_{a}^{n}}^{2}}{\left|u^{n}-a\right|_{\mathcal{P}_{a}^{n}}^{2}} .
$$

The analogous condition to (4.18) with $\xi_{a}^{n}$ replaced by

$$
\xi_{b}^{n}=1-\frac{|b-a|_{\mathcal{P}_{b}^{n}}^{2}}{\left|u^{n}-b\right|_{\mathcal{P}_{b}^{n}}^{2}}
$$


has to hold on $\mathcal{P}_{b}^{n}$. This implies that there exist $\sigma>\alpha$ being feasible for condition (4.17). In view of $\rho+\alpha \leq \sigma$ for some $\rho>0$ we must have $\sigma>\alpha$. Hence (4.17) and the first condition of Corollary 4.6 are not contradictory.

Since we are interested in the sequence of iterates, we additionally have to prove that

$$
\xi^{n}=\max \left\{\xi_{a}^{n}, \xi_{b}^{n}\right\}
$$

is uniformly bounded away from 1 . This is the content of the following lemma.

LEMma 4.10. Suppose that

$\operatorname{essinf}\left\{b(x)-a(x) \mid x \in \mathcal{P}^{n}\right\} \geq \underline{\epsilon}>0 \quad$ for all $n \in \mathbb{N} . \quad\left(\operatorname{essinf}=+\infty\right.$ if $\left.\operatorname{meas}\left(P^{n}\right)=0.\right)$

Then there exists $0 \leq \bar{\xi}<1$ such that $0 \leq \xi^{n} \leq \bar{\xi}$ for all iteration levels $n$.

Proof. We will only argue for $\xi_{a}^{n}$ since similar arguments apply for $\xi_{b}^{n}$.

First observe that (4.15a) implies $p^{n} \in C(\bar{\Omega})$ by Sobolev's Embedding Theorem. Consequently, the bound

$$
\left|u^{n}-a\right|_{\mathcal{P}_{a}^{n}} \leq\left(\beta_{u_{d}}+\alpha^{-1} \beta_{p}|\tau|+\beta_{a}\right) \sqrt{\operatorname{meas}\left(\mathcal{P}_{a}^{n}\right)}
$$

is valid, where the existence of the uniform bounds $\beta_{a}, \beta_{u_{d}}, \beta_{p}>0$ is due to $u_{d}, a \in$ $L^{\infty}\left(\Gamma_{1}\right)$ and $p^{n} \in C(\bar{\Omega})$ and the uniform boundedness of $p^{n}$ in $H^{1}(\Omega)$. On the other hand, due to our assumption we obtain

$$
|b-a|_{\mathcal{P}_{a}^{n}} \geq \underline{\epsilon} \sqrt{\operatorname{meas}\left(\mathcal{P}_{a}^{n}\right)} \quad \text { for all } n \in \mathbb{N} .
$$

Therefore,

$$
\xi_{a}^{n}=1-\frac{|b-a|_{\mathcal{P}_{a}^{n}}^{2}}{\left|u^{n}-a\right|_{\mathcal{P}_{a}^{n}}^{2}} \leq 1-\frac{\epsilon^{2}}{M_{a}^{2}}=: \bar{\xi}_{a}<1
$$

with $M_{a}=\beta_{u_{d}}+\alpha^{-1} \beta_{p}|\tau|+\beta_{a}$. In the same manner, we obtain $\xi_{b}^{n} \leq \bar{\xi}_{b}<1$.

Taking $\bar{\xi}=\max \left\{\bar{\xi}_{a}, \bar{\xi}_{b}\right\}<1$ completes the proof.

To proceed in our discussion of the conditions on $\alpha$ and $\sigma$, we consider $\alpha+\alpha^{2} C^{-2}$ $\alpha^{2} \rho^{-1}, \rho>0$, the upper bound on $\sigma$ in the first condition of Corollary 4.6. Since we have $\sigma \geq \alpha+\rho>0$ by the lower bound, we obtain

$$
\rho=\gamma \frac{\alpha C^{2}}{C^{2}+\alpha}, \quad \gamma>1
$$

Inserting this expression for $\rho$ in

$$
\rho+\alpha<\alpha+\frac{\alpha^{2}}{C^{2}}-\frac{\alpha^{2}}{\rho}
$$

and putting $z=\rho / \alpha$, one obtains the following condition as a result:

$$
z<d-\frac{1}{z} \quad \text { with } d=\frac{\alpha}{C^{2}} .
$$

Consequently, for $\alpha>2 C^{2}$, there exist $z \in \mathbb{R}_{+}$satisfying (4.21). Fig. 2 gives a graphical illustration. The curve for $d_{2}$ corresponds to $\alpha=2 C^{2}$, i.e., $d_{2}=2$, while $d_{1}>2$ and $d_{3}<2$. 


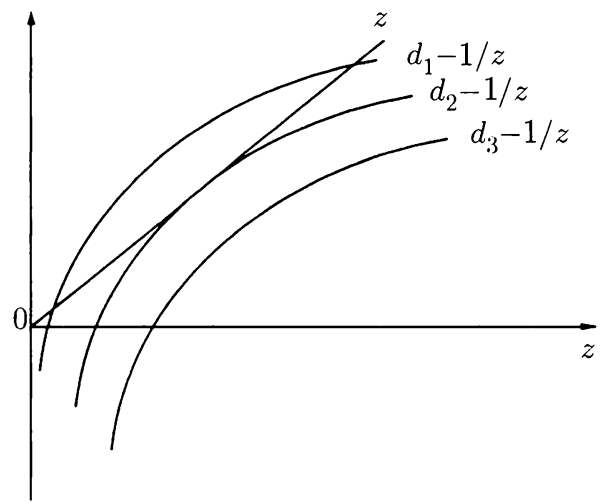

FIG. 2. Condition (4.21)

It remains to combine both conditions of Corollary 4.6. Considering the uniform bound on $\xi^{n}$, condition (4.18) becomes

$$
\sigma \bar{\xi} \leq \alpha
$$

We assume that $C$ is sufficiently large. Then (4.22) and $\rho+\alpha \leq \sigma$ yield

$$
\bar{\xi}(\rho+\alpha) \leq \bar{\xi} \sigma<\alpha
$$

This implies

$$
\gamma \frac{\alpha C^{2}}{\alpha+C^{2}} \leq \frac{1-\bar{\xi}}{\bar{\xi}} \alpha .
$$

Suppose now that $\alpha=q C^{2}, q>2$. From the inequality above, we deduce

$$
\gamma \leq \frac{(1-\bar{\xi})\left(\alpha+C^{2}\right)}{C^{2} \bar{\xi}}=\frac{(q+1)(1-\bar{\xi})}{\bar{\xi}} .
$$

From (4.20) we know that $\gamma>1$ has to be satisfied. Hence, for our choice of $\alpha$, the condition $\bar{\xi}<(q+1) /(q+2)$ must hold.

A final observation concerning the parameter $\rho$ is the fact that

$$
\rho=\alpha \text { maximizes the length of the interval }\left[\rho+\alpha, \alpha+\frac{\alpha^{2}}{C^{2}}-\frac{\alpha^{2}}{\rho}\right] .
$$

5. The finite-dimensional algorithm. For implementation reasons, one must discretize the infinite-dimensional problem (1.1) by finite differences or finite elements. After discretization, the quadratic programming problem denoted by

$$
\begin{array}{ll}
\text { minimize } & Q(Y, U):=\frac{1}{2}\left\|M_{\Omega}^{1 / 2}\left(Y-Z_{d}\right)\right\|_{k}^{2}+\frac{\alpha}{2}\left\|M_{\Gamma}^{1 / 2}\left(U-U_{d}\right)\right\|_{l}^{2} \\
\text { subject to } & S_{\Omega} Y=G-S_{\Gamma} U \\
\text { and } & A \leq U \leq B
\end{array}
$$

is obtained. In (5.1) we used $Y, Z_{d}, G \in \mathbb{R}^{k}$ and $U, U_{d}, A, B \in \mathbb{R}^{l}$. The matrices $M_{\Omega} \in$ $\mathbb{R}^{k \times k}$ and $M_{\Gamma} \in \mathbb{R}^{l \times l}$ are symmetric and positive definite, the matrix $S_{\Omega} \in \mathbb{R}^{k \times k}$ is 
symmetric and nonsingular, and $S_{\Gamma} \in \mathbb{R}^{k \times l}$. The norms $\|\cdot\|_{k}$ and $\|\cdot\|_{l}$ denote the Euclidean norms of $\mathbb{R}^{k}$ and $\mathbb{R}^{l}$, respectively. For the corresponding inner product, we use $(w, z)_{k}=w^{\mathrm{T}} v$ in $\mathbb{R}^{k}$ and analogously for $\mathbb{R}^{l}$. By $w_{\mid S}$ we denote the components $w_{i}$ with $i \in S \subset\{1, \ldots, l\}=: \mathbb{N}_{\Gamma}$. The norm $\|w\|_{S}$ is defined as $\|\bar{w}\|_{l}$ with $\bar{w}_{\mid S}=w_{\mid S}$ and $\bar{w}_{\mid S^{\prime}}=0$, where $S^{\prime}=\mathbb{N}_{\Gamma} \backslash S$.

As an immediate consequence of our assumptions, we can ensure the existence of the unique solution $\left(Y^{*}, U^{*}\right) \in \mathbb{R}^{k} \times \mathbb{R}^{l}$ of (5.1) characterized by the following first-order conditions:

$$
\begin{aligned}
M_{\Omega}\left(Y^{*}-Z_{d}\right)+S_{\Omega} P^{*} & =0 \\
\alpha M_{\Gamma}\left(U^{*}-U_{d}\right)+S_{\Gamma}^{T} P^{*}+\Lambda^{*} & =0 \\
S_{\Omega} Y^{*}+S_{\Gamma} U^{*}-G & =0 \\
\Lambda^{*} & =\sigma\left[U^{*}+\sigma^{-1} \Lambda^{*}-\Pi_{U_{\text {ad }}}\left(U^{*}+\sigma^{-1} \Lambda^{*}\right)\right],
\end{aligned}
$$

where $P^{*} \in \mathbb{R}^{k}, \Lambda^{*} \in \mathbb{R}^{l}$ denote the Lagrange multipliers, and $\Pi_{U_{\text {ad }}}$ stands for the componentwise projection onto $U_{\text {ad }}:=\left\{i \in \mathbb{N}_{\Gamma} \mid A_{i} \leq U_{i} \leq B_{i}\right\}$. The discretized active and inactive sets are defined by

$$
\begin{aligned}
& A_{a}^{n}=\left\{i \in \mathbb{N}_{\Gamma} \mid U_{i}^{n-1}+\sigma^{-1} \Lambda_{i}^{n-1}<A_{i}\right\}, \\
& A_{b}^{n}=\left\{i \in \mathbb{N}_{\Gamma} \mid U_{i}^{n-1}+\sigma^{-1} \Lambda_{i}^{n-1}>B_{i}\right\}, \\
& A^{n}=A_{a}^{n} \cup A_{b}^{n} \text { and } I^{n}=\mathbb{N}_{\Gamma} \backslash A^{n} .
\end{aligned}
$$

The finite-dimensional version of Algorithm AS becomes

\section{Algorithm AS_finite}

Output: exact optimal solution $\left(Y^{*}, U^{*}\right)$.

\section{begin}

$\left(Y^{o}, U^{o}, P^{o}, \Lambda^{o}\right) \leftarrow$ initialization $\left(Z_{d}, G, U_{d}, A, B, M_{\Omega}, M_{\Gamma}, S_{\Omega}, S_{\Gamma}, \alpha\right)$

$n \leftarrow 0$

end

while $\left(A^{n} \neq A^{n-1}\right)$ or $(n<2)$

Compute $A_{a}^{n}, A_{b}^{n}$ and set $A^{n} \leftarrow A_{a}^{n} \cup A_{b}^{n}, I_{n} \leftarrow \mathbb{N}_{\Gamma} \backslash A^{n}$.

Determine $\left(Y^{n}, U^{n}, P^{n}, \Lambda^{n}\right)$ such that

$$
\begin{aligned}
M_{\Omega}\left(Y^{n}-Z_{d}\right)+S_{\Omega} P^{n} & =0, \\
\alpha M_{\Gamma}\left(U^{n}-U_{d}\right)+S_{\Gamma}^{T} P^{n}+\Lambda^{n} & =0, \\
S_{\Omega} Y^{n}+S_{\Gamma} U^{n}-G & =0
\end{aligned}
$$

end

with $U_{\mid A_{a}^{n}}^{n} \leftarrow A_{\mid A_{a}^{n}}, U_{\mid A_{b}^{n}}^{n} \leftarrow B_{\mid A_{b}^{n}}$, and $\Lambda_{\mid I^{n}} \leftarrow 0$.

The identification $A^{n}=A^{n-1}$ is understood in the (discretized) sense of (3.1). An example for a simple initialization strategy is the following procedure ( $\mathrm{min}$ is understood in the componentwise sense). In parentheses we denote an alternative.

\section{initialization}

Input: $Z_{d}, G, U_{d}, A, B, M_{\Omega}, M_{\Gamma}, S_{\Omega}, S_{\Gamma}, \alpha$.

Output: $Y^{o}, U^{o}, P^{o}, \Lambda^{o}$.

begin 
$U^{o} \leftarrow A .\left(U^{o} \leftarrow B\right)$

Compute $Y^{o}$ and $P^{o}$ as solution to

$$
\begin{gathered}
S_{\Omega} Y=G-S_{\Gamma} U^{o}, \\
S_{\Omega} P+M_{\Omega} Y=M_{\Omega} Z_{d} \\
\text { end }
\end{gathered}
$$

The convergence analysis of Algorithm AS_finite is based on the same ideas as in the infinite-dimensional context. We make use of the discrete analogue of the modified augmented Lagrangian function of the previous section:

$$
\begin{aligned}
\bar{L}\left(Y^{n}, U^{n}, \Lambda^{n}\right)=Q\left(Y^{n}, U^{n}\right)+\frac{1}{2 \sigma}[(\|(\sigma(A & \left.\left.\left.-U^{n}\right)-\Lambda_{-}^{n}\right)_{+}\left\|_{S_{a}^{n}}^{2}-\right\| \Lambda_{-}^{n} \|_{S_{a}^{n}}^{2}\right) \\
& \left.+\left(\left\|\left(\sigma\left(U^{n}-B\right)+\Lambda_{+}^{n}\right)_{+}\right\|_{S_{b}^{n}}^{2}-\left\|\Lambda_{+}^{n}\right\|_{S_{b}^{n}}^{2}\right)\right],
\end{aligned}
$$

where $(\cdot)_{+}$is understood componentwise, and $S_{a}^{n}=I^{n} \cup A_{a}^{n}$, analogously for $S_{b}^{n}$.

The following convergence theorem states that Algorithm AS_finite stops after a finite number of iterations in the exact solution of problem (5.1). We denote by $\underline{\lambda}>0$ the smallest and by $\bar{\lambda}$ the largest eigenvalue of $M_{\Gamma}$. The set $\overline{\mathcal{P}}^{n}$ is the discrete analogue of $\mathcal{P}^{n}$ of Sec. 4 .

Theorem 5.1. Suppose that $A^{n} \neq A^{n-1}$ and $\inf \left\{B_{i}-A_{i} \mid i \in \overline{\mathcal{P}}^{n}\right\} \geq \epsilon>0$ hold. If

$$
\alpha \bar{\lambda}+\rho \leq \sigma<\underline{\lambda} \alpha+\frac{\alpha^{2}}{c_{2}^{2}}-\frac{c_{1}^{2} \alpha^{2}}{\rho c_{2}^{2}} \quad \text { and } \quad(\sigma-\alpha \underline{\lambda})\left\|U^{n}-U^{n-1}\right\|_{\overline{\mathcal{P}}^{n}}^{2}-\sigma\|A-B\|_{\overline{\mathcal{P}}^{n}}^{2} \leq 0
$$

for some $\rho>0$, then

$$
\bar{L}\left(Y^{n}, U^{n}, \Lambda^{n}\right)-\bar{L}\left(Y^{n-1}, U^{n-1}, \Lambda^{n-1}\right)<0,
$$

where $c_{1}=\left\|S_{\Gamma}^{T} S_{\Omega}^{-1} M_{\Omega}^{1 / 2}\right\|$ and $c_{2}=\left\|M_{\Gamma}^{-1} S_{\Gamma}^{T} S_{\Omega}^{-1} M_{\Omega}^{1 / 2}\right\|$. Moreover, if (5.2) holds for all $n \geq \bar{n}, \bar{n} \in \mathbb{N}$, then Algorithm AS_finite stops after a finite number of iterations at the exact solution $\left(Y^{*}, U^{*}\right)$ of $(5.1)$.

Proof. First observe that if there exists an iteration level $n$ such that $A^{n-1}=A^{n}$, then the discrete algorithm stops at the exact solution.

Next we establish the descent property of the discrete modified augmented Lagrangian function. We will only display the major steps since the ideas and computations are similar to the infinite-dimensional case. For convenience we define $\Delta_{Y}^{n}=Y^{n}-Y^{n-1}$ and $\Delta_{U}^{n}=U^{n}-U^{n-1}$. We have

$$
Q\left(Y^{n}, U^{n}\right)-Q\left(Y^{n-1}, U^{n-1}\right)=-\frac{1}{2}\left\|M_{\Omega}^{1 / 2} \Delta_{Y}^{n}\right\|_{k}^{2}-\frac{\alpha}{2}\left\|M_{\Gamma}^{1 / 2} \Delta_{U}^{n}\right\|_{l}^{2}-\left(\Delta_{U}^{n}, \Lambda^{n}\right)_{l}
$$

and the following estimate for the inner product:

$$
\left(-\Delta_{U}^{n}, \Lambda^{n}-\Lambda^{n-1}\right)_{l} \leq \alpha\left\|M_{\Gamma}^{1 / 2} \Delta_{U}^{n}\right\|_{A_{*}^{n}}^{2}+c_{1}\left\|\Delta_{U}^{n}\right\|_{A_{*}^{n}}\left\|M_{\Omega}^{1 / 2} \Delta_{Y}^{n}\right\|_{k},
$$

where $A_{*}^{n}$ is the discrete analogue of $\mathcal{A}_{*}^{n}$. On $I_{*}^{n}$, the discrete analogue of $\mathcal{I}_{*}^{n}$, we have

$$
\left\|\Delta_{U}^{n}\right\|_{I_{*}^{n}} \leq \frac{c_{2}}{\alpha}\left\|M_{\Omega}^{1 / 2} \Delta_{Y}^{n}\right\|_{k}
$$


Therefore, the following estimate is valid:

$$
\begin{aligned}
\bar{L}\left(Y^{n}, U^{n}, \Lambda^{n}\right)-\bar{L}\left(Y^{n-1}, U^{n-1}, \Lambda^{n-1}\right) \leq \frac{1}{2} & \left(\frac{\sigma-\underline{\lambda} \alpha}{\alpha^{2}} c_{2}^{2}+\frac{c_{1}^{2}}{\rho}-1\right)\left\|M_{\Omega}^{1 / 2} \Delta_{Y}^{n}\right\|_{k}^{2} \\
& +\frac{1}{2}(\alpha \bar{\lambda}+\rho-\sigma)\left\|\Delta_{U}^{n}\right\|_{A_{*}^{n}}^{2} \\
& +\frac{1}{2}(\sigma-\underline{\lambda} \alpha)\left\|\Delta_{U}^{n}\right\|_{\overline{\mathcal{P}}^{n}}^{2}-\frac{\sigma}{2}\|A-B\|_{\overline{\mathcal{P}}^{n}}^{2},
\end{aligned}
$$

with $\rho=\kappa c_{1}$. Hence, condition (5.2) is sufficient for $\bar{L}\left(Y^{n}, U^{n}, \Lambda^{n}\right)-\bar{L}\left(Y^{n-1}, U^{n-1}, \Lambda^{n-1}\right)$ $<0$ for all $n \in \mathbb{N}$.

Finally, since there exists only a finite number of possible active and inactive sets, and we have strict descent of the discrete modified augmented Lagrangian function under (5.2), Algorithm AS_finite has to stop after a finite number of iterations.

Note that (5.2) also states a condition for the discretization of the control space, since the eigenvalues of $M_{\Gamma}$ are involved.

REMARK 5.2. If one considers the specific discretization with $M_{\Omega}=h^{2} E_{k}$ and $M_{\Gamma}=$ $h E_{l}$, where $E_{k}, E_{l}$ denote the unit matrices of $\mathbb{R}^{k \times k}$ and $\mathbb{R}^{l \times l}$, respectively, and $h$ is the mesh-size, then $\bar{\lambda}=h, \underline{\lambda}=h$, and $c_{1}=h\left\|S_{\Gamma}^{T} S_{\Omega}^{-1}\right\|=h c_{2}=: h^{1 / 2} C_{h}$. Hence, condition (5.2) becomes

$$
\alpha+\hat{\rho} \leq \hat{\sigma}<\alpha+\frac{\alpha^{2}}{C_{h}^{2}}-\frac{\alpha^{2}}{\hat{\rho}} \quad \text { and } \quad(\hat{\sigma}-\alpha)\left\|\Delta_{U}^{n}\right\|_{\overline{\mathcal{P}}^{n}}^{2}-\hat{\sigma}\|A-B\|_{\overline{\mathcal{P}}^{n}}^{2} \leq 0,
$$

with $\hat{\rho}=h^{-1} \rho, \hat{\sigma}=h^{-1} \sigma$, which is the discrete analogue of condition (4.10) of Corollary 4.6 and can be analyzed similarly.

6. Numerical tests. We shall now report on numerical testing of Algorithm AS_finite on a DECAlpha 500 workstation with machine precision $\varepsilon_{M} \approx 1.1 \cdot 10^{-16}$. The implementation was done in MATLAB 5.2. We will display the results for a representative selection of test examples. The domain $\Omega$ was chosen to be $\Omega=(0,1)^{2}$. In all tests below, the control was taken to act on $\Gamma_{1}=(0,1) \times\{0\}$. The discretization of the Laplacian was realized by the five-point star. The discretization of the normal derivative was based on symmetric differences. Moreover, for numerical integration, i.e., discretization of the objective functional, we chose $h^{-2} M_{\Omega}$ and $h^{-1} M_{\Gamma}$ (compare (5.1)) to be the unit matrices of the respective finite-dimensional space. Therefore, considering Remark 5.2, the constant $C_{h}$ appearing in the sufficient conditions for descent of the modified augmented Lagrangian function becomes $C_{h}=h^{1 / 2}\left\|S_{\Gamma}^{T} S_{\Omega}^{-1}\right\|$. Unless otherwise specified, the mesh-size was $h=1 / 50$ and the initialization with $U^{o}=B$ was used.

6.1. Test examples. In all test examples specified below, we used $g \equiv 0$ for convenience.

EXAMPLE 6.1.

Bounds: $a \equiv-0.75, b \equiv 0.75$. Desired state: $z_{d}=\frac{1}{6} \sin \left(2 \pi x_{1}\right) \sin \left(2 \pi x_{2}\right) e^{2 x_{1}}$. Desired control: $u_{d}=\cos \left(5 \pi x_{1}^{2}\right)$. Potential term: $c \equiv 1$. Parameter values: $\alpha=10^{-3}, \hat{\sigma}=10^{-1}$. Remark: The example is constructed such that there exist several active and inactive regions in the interior and on the boundary of $\Gamma_{1}$. Moreover, both bounds are active at the optimal (numerical) solution. Table 1 displays the values for $C_{h}=h^{1 / 2}\left\|S_{\Gamma}^{T} S_{\Omega}^{-1}\right\|$ for several mesh-sizes $h$. 


\begin{tabular}{|c||c|c|c|c|c|}
\hline$h$ & $10^{-1}$ & $20^{-1}$ & $30^{-1}$ & $40^{-1}$ & $50^{-1}$ \\
\hline$C_{h}$ & 0.02634 & 0.00959 & 0.00526 & 0.00342 & 0.00245 \\
\hline
\end{tabular}

TABLE 1. Values for $C_{h}=h^{1 / 2}\left\|S_{\Gamma}^{T} S_{\Omega}^{-1}\right\|$ for varying $h$

\begin{tabular}{|c|c|c|c|c|c|}
\hline$r^{n}$ & $\left|A_{a}^{n}\right|$ & $\left|A_{b}^{n}\right|$ & $\left|\overline{\mathcal{P}}^{n}\right|$ & $J^{n}$ & $\bar{L}^{n}$ \\
\hline $2.4920 e-1$ & 0 & 69 & 0 & $4.225353 e-2$ & $8.939324 e-2$ \\
\hline 0.0000 & 34 & 69 & 0 & $4.225588 e-2$ & $4.225588 e-2$ \\
\hline 0.0000 & 34 & 69 & 0 & $4.225588 e-2$ & $4.225588 e-2$ \\
\hline \multicolumn{7}{|c|}{ TABLE 2. Performance for Example 6.1 }
\end{tabular}

\section{EXAMPLE 6.2.}

Bounds: $a \equiv-0.05, b \equiv 0.05$. Desired state: $z_{d}=\frac{1}{6} \sin \left(2 \pi x_{1}\right) \sin \left(2 \pi x_{2}\right) e^{2 x_{1}}$. Desired control: $u_{d} \equiv 0$. Potential term: $c \equiv 1$. Parameter values: $\hat{\sigma}=10^{-1}$, several values for $\alpha$ are tested.

EXAMPLE 6.3.

Bounds: $a\left(x_{1}\right)=-0.1\left|\sin \left(6 \pi x_{1}\right)\right|, b\left(x_{1}\right)=2+\cos \left(11 \pi x_{1}\right)$ for $0<x_{1}<1$. Desired state:

$$
z_{d}=\left\{\begin{aligned}
0 & \text { for } x_{1} \leq \frac{1}{2} \\
1 & \text { for } \frac{1}{2}<x_{1} \leq \frac{3}{4} \\
-1 & \text { for } x_{1}>\frac{3}{4}
\end{aligned}\right.
$$

Desired control: $u_{d} \equiv 0$. Potential term: $c=10 x_{1}+100 x_{2}$. Parameter values: $\alpha=10^{-5}$, $\hat{\sigma}=10^{-2}$. Remark: The example is constructed such that $B$ is nowhere active at the optimal solution. The choice of $B$ yields an oscillating initial value for $U$ if the algorithm is started with $U^{o}=B$. For $h=1 / 50$ we obtain $C_{h}=0.00245$.

EXAMPLE 6.4.

Bounds: $a\left(x_{1}\right)=\sin \left(8 \pi x_{1}\right), b\left(x_{1}\right)=2+\cos \left(\frac{\pi}{2}+8 \pi x_{1}\right)$ for $0<x_{1}<1$. The desired state and control are chosen as in Example 6.3. Potential term: $c \equiv 1$. Parameter values: $\alpha=10^{-4}, \hat{\sigma}=10^{-2}$. Remark: The bounds are chosen such that there exist $x_{1} \in(0,1)$ with $a\left(x_{1}\right)=b\left(x_{1}\right)$ and such that meas $\left\{x_{1} \in(0,1) \mid a\left(x_{1}\right)=b\left(x_{1}\right)\right\}=0$.

6.2. Results. Table 2 gives the results for a run of Algorithm AS_finite for Example 6.1 and $h=1 / 200$. By $\left|S^{n}\right|, S^{n} \subset \mathbb{N}_{\Gamma}$, we denote the number of components $i$ of $U^{n}$ with $i \in S^{n}$. Thereby $r^{n}$ is the maximal violation of the bound constraints. If we extrapolate Table 1, then $\alpha=10^{-3}$ satisfies $\alpha>2 C_{h}^{2}$ (see the discussion in $\S 4$ ) for $h \leq 1 / 20$. The algorithm also exhibits a descent behavior of $\bar{L}^{n}$ for $h=1 / 10$. Moreover, for all $h_{i}=1 / i, i=10,20, \ldots, 200$, only two iterations are needed until the algorithm stops with the optimal discrete solution. This mesh-independent behavior for control constrained examples is also observed in [4] for distributed and unilaterally constrained optimal control problems. The low number of iterations is typical for most of the test runs.

Next we test Example 6.2 for various values of $\alpha$. From Table 1 we know that $\alpha>$ $1.1972 \cdot 10^{-5}$ has to be satisfied for the sufficient conditions (see Theorem 5.1). Indeed, 


\begin{tabular}{|c|c|c|c|c|c|}
\hline$r^{n}$ & $\left|A_{a}^{n}\right|$ & $\left|A_{b}^{n}\right|$ & $\left|\overline{\mathcal{P}}^{\prime \prime}\right|$ & $J^{n}$ & $\bar{L}^{n}$ \\
\hline $3.6086 \mathrm{c}+1$ & 0 & 27 & 0 & $4.192950 e-2$ & $4.334799 e+2$ \\
\hline 0.0000 & 20 & 29 & 0 & $4.223890 e-2$ & $4.223890 e-2$ \\
\hline 7.7727 & 20 & 27 & 2 & $4.223668 e-2$ & 3.691441 \\
\hline 0.0000 & 22 & 27 & 0 & $4.223884 e-2$ & $4.223884 e-2$ \\
\hline 0.0000 & 22 & 27 & 0 & $4.223884 e-2$ & $4.223884 e-2$ \\
\hline
\end{tabular}

TABLE 3. Example 6.2 for $\alpha=10^{-6}$

for $\alpha \leq 10^{-5}$ the following behavior displayed in Table 3 can be observed. Although the algorithm stops at the exact solution, the merit function is not strictly decreasing. Since $\overline{\mathcal{P}}^{n} \neq \varnothing$ for $n=3$ we shall further analyze the dependence on $\hat{\sigma}$. The second condition of Theorem 5.1, which involves $\overline{\mathcal{P}}^{n} \neq \varnothing$, also depends on the difference $\hat{\sigma}-\alpha$. Hence, we reduce $\hat{\sigma}$ to $\hat{\sigma}=1.1 \cdot 10^{-6}$ (for $\alpha=10^{-6}$ ), and find that $\bar{L}^{n}$ exhibits a descent behavior in each iteration. Note that, due to our initialization, i.e., $U^{o}=B$, the choice of $\hat{\sigma}$ has no influence on the sequence of iterates. It only guarantees that $\bar{L}^{n}$ is strictly decreasing. In Fig. 3 we give plots for the optimal control $U^{*}$ and the corresponding multiplier $\Lambda^{*}$ for $h=1 / 50$ and $\alpha=10^{-6}$. The left graph of Fig. 3 shows a "bang-bang" control, which is the result of the increasing singularity of the problem for decreasing $\alpha$. Hence, there is a switching point where $U^{*}$ jumps from the lower bound to the upper bound. Consequently, the inactive set at the optimal solution is empty.
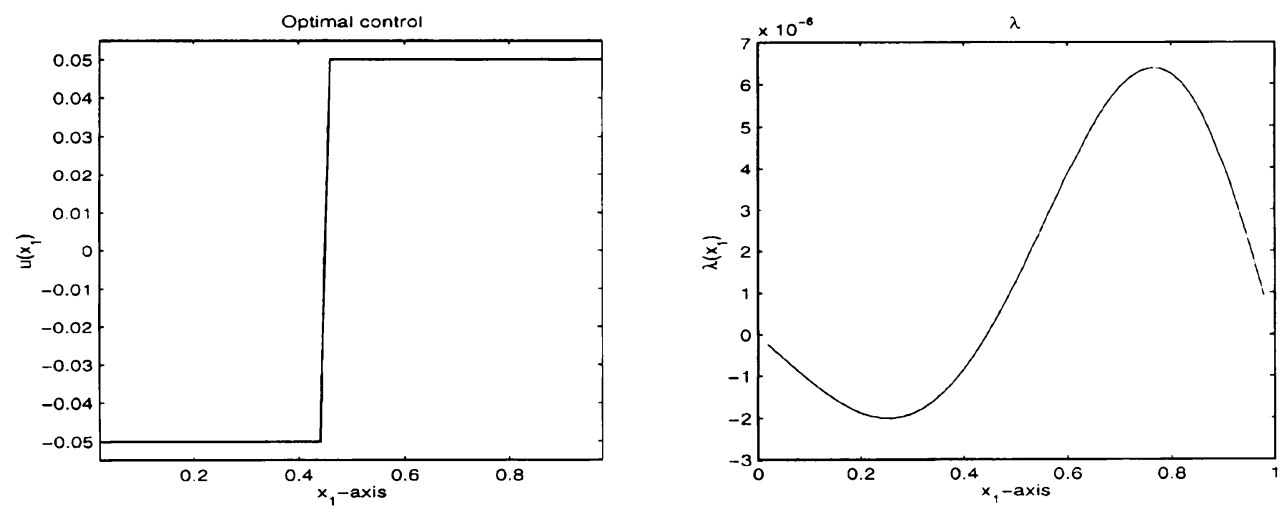

FIG. 3. Optimal state and multiplier for Example 6.2 with $\alpha=10^{-6}$

Now we turn to Example 6.3. The aim of the construction is twofold: first, we want to test the influence of the nonconstant $c$; secondly, only the lower bound is active at the optimal solution, and the iteration process is started with $U^{o}$ set to the "wrong" bound which, in addition, is rather oscillatory. Let us give the plots of the optimal state, adjoint state, control and multiplier in Fig. 4. The algorithm stops after two iterations at the exact (discrete) solution. This behavior is again independent of the mesh-size. A reduction of $\alpha$ to $\alpha=10^{-5}$, which clearly violates $\alpha>2 C_{h}^{2}$, yields the result of Table 4. Now there is also a part of $B$ that is active at the optimal solution. Although $\alpha$ 

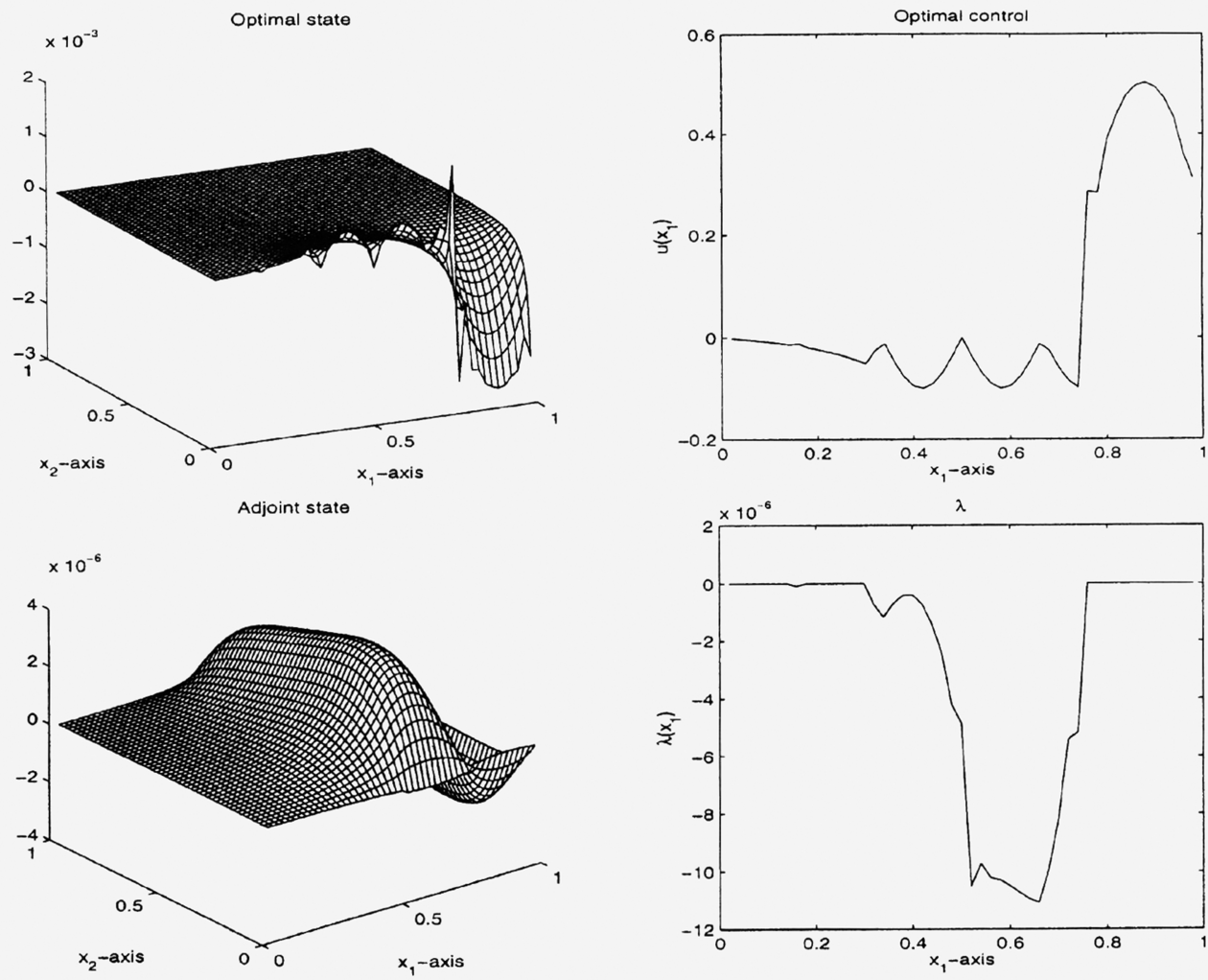

FIG. 4. Plots of the optimal solution of Example 6.3

\begin{tabular}{|c|c|c|c|c|c|}
\hline$r^{n}$ & $\left|A_{a}^{n}\right|$ & $\left|A_{b}^{n}\right|$ & $\left|\overline{\mathcal{P}}^{n}\right|$ & $J^{n}$ & $\bar{L}^{n}$ \\
\hline $5.1502 e+1$ & 0 & 12 & 0 & $2.366900 e-1$ & $1.276744 e+3$ \\
\hline 0.0000 & 37 & 12 & 0 & $2.397982 e-1$ & $2.397982 e-1$ \\
\hline 0.0000 & 37 & 12 & 0 & $2.397982 e-1$ & $2.397982 e-1$ \\
\hline \multicolumn{7}{|c|}{ TABLE 4. Example 6.3 for $\alpha=10^{-5}$}
\end{tabular}

is less than $2 C_{h}^{2}$, the modified augmented Lagrangian function is decreasing. Moreover, the identification and correction process is very efficient again. Let us remark that we also tested the case where $c \equiv 0$. The constants of Table 1 change only slightly (in the last digit). In all cases, i.e., $h_{i}=1 / i, i=10, \ldots, 50$, the algorithm stopped after two iterations at the exact (discrete) solution.

We consider Example 6.4. There exist points $x_{1}$ such that the Slater condition, i.e., the interior of $U_{a d}$ is nonempty, does not hold. Since meas $\left\{x_{1} \in(0,1) \mid a\left(x_{1}\right)=b\left(x_{1}\right)\right\}=0$, the lack of the Slater condition is benign. The mesh-size $h$ was chosen such that there exist components $i$ with $A_{i}=B_{i}$ numerically. Again, the algorithm terminates after two iterations at the exact (discrete) solution. Fig. 5 displays the optimal control (together with the bounds $A$ and $B$ ) and the corresponding multiplier for $h=1 / 100$. 

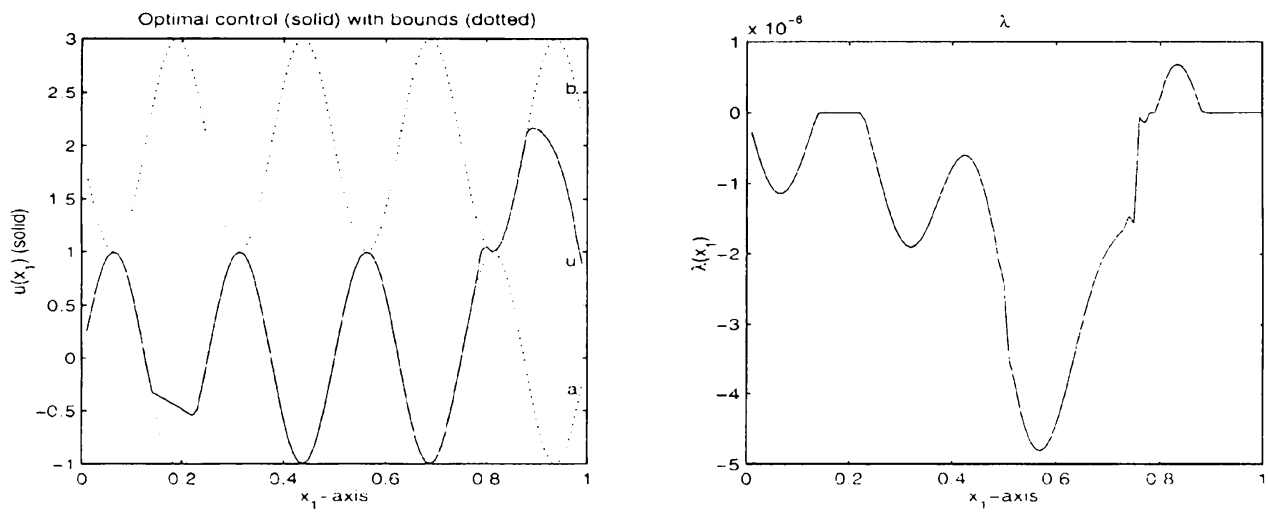

FIG. 5. Plots of the optimal solution of Example 6.4

Finally, we consider the aspect of lack of strict complementarity, i.e., there exists $i \in A^{*}$ such that $\lambda_{i}^{*}=0$. Sometimes this fact is referred to as degeneracy of the problem. It is known for the methods based on gradient projection (see, for instance, [20]) that their performance degrades with increasing degree of degeneracy. Since the sequence of iterates of Algorithm AS_finite depends on the sign of $\Lambda_{i}^{n}$ for $i \in A^{n}$ and the sign of $U_{i}^{n}-B_{i}$, respectively $U_{i}^{n}-A_{i}$, for $i \in I^{n}$, and since the precision of $\Lambda^{n}, U^{n}$ essentially depends on the condition number of $S_{\Omega}$ and round-off errors, the active and inactive sets may start to chatter near the optimal solution with lack of strict complementarity. Therefore, we introduce an additional stopping criterion, which is similar to a rule developed in [4]. For this purpose define

$$
S^{n}=\left\{i \in A_{b}^{n} \mid \Lambda_{i}^{n} \leq 0\right\} \cup\left\{i \in A_{a}^{n} \mid \Lambda_{\imath}^{n} \geq 0\right\} \quad \text { and } \quad T^{n}=\left\{i \in I^{n} \mid\left(U_{i}^{n}>B_{i}\right) \vee\left(U_{i}^{n}<A_{i}\right)\right\}
$$

and

$$
r_{S}^{n}=\max _{i \in S^{n}}\left\{\left|\Lambda_{i}^{n}\right|\right\} \quad \text { and } \quad r_{T}^{n}=\max _{i \in T^{n}}\left\{\min \left\{\left|U_{i}^{n}-B_{i}\right|,\left|U_{i}^{n}-A_{i}\right|\right\}\right\} .
$$

If we find that $r_{S}^{n}$ and $r_{T}^{n}$ are of the order of the precision expected for the solution of the linear system that has to be solved in each iteration of Algorithm AS_finite, we cannot rely on the determination of active and inactive sets. Hence we stop the algorithm. We constructed examples with lack of strict complementarity at the exact solution. The algorithm immediately detects the correct active and inactive sets and terminates after one iteration.

7. Conclusion. Algorithms for solving optimal control problems with inequality constraints on the control are still a significant challenge. In this paper we have developed an efficient, easy to implement algorithm for a representative class of control constrained optimal control problems. We have proved global convergence, i.e., convergence from any starting point to the optimal solution, in finite dimensions and in infinite dimensions under certain conditions on the parameters involved, respectively. In finite dimensions, the algorithm has a finite termination property. Based on a very well suited identification 
strategy for the active and inactive sets, which allows multiple activation and inactivation of constraints, in the discrete case the new algorithm proved to be very competitive in practice, exhibits a low number of iterations needed to find the exact optimal solution and turns out to be very robust even in the case of lack of strict complementarity.

\section{Appendix A.}

Proof of Lemma 2.1. Define the bilinear form $a: V \times V \rightarrow \mathbb{R}$ by

$$
a(w, v)=(\nabla w, \nabla v)_{\Omega}+(c w, v)_{\Omega} .
$$

From $\bar{c} \geq c \geq 0$ and the Poincaré-Friedrichs Inequality, we immediately find $a$ to be $V$-elliptic and bounded. Next, we define the linear functional $f: V \rightarrow \mathbb{R}$ by

$$
f(v)=(g, v)_{\Omega}+\left(u, \tau v_{\mid \Gamma_{1}}\right)_{\Gamma_{1}} .
$$

The continuity of $f$ follows from the continuity of the trace map $\tau: H^{1}(\Omega) \rightarrow L^{2}(\Gamma)$.

By the Lax Milgram Theorem, the variational problem

$$
a(y, v)=f(v) \quad \text { for all } v \in V
$$

admits a unique solution $y^{s} \in V$.

From (A.1) we immediately detect $-\Delta y+c y=g$ in the weak sense and $y=0$ on $\Gamma_{2}$ by the definition of $V$. Define

$$
H^{1}(\Delta, \Omega)=\left\{w \in H^{1}(\Omega) \mid \Delta w \in L^{2}(\Omega)\right\}
$$

endowed with the norm

$$
|w|_{H^{1}(\Delta, \Omega)}=\left(|u|_{H^{1}(\Omega)}^{2}+|\Delta u|_{\Omega}^{2}\right)^{1 / 2} .
$$

Essentially following the arguments of [5], we find that the trace mapping $\tau_{\Delta}: H^{1}(\Delta, \Omega) \rightarrow$ $H^{-1 / 2}(\Gamma), \tau_{\Delta} w=\left.\frac{\partial w}{\partial n}\right|_{\Gamma}$ is continuous and that the generalized Green's formula holds:

$$
\left\langle\frac{\partial y}{\partial n}, \tau v\right\rangle=\int_{\Omega}(\Delta y-c y) v d x+a(y, v)
$$

for all $y \in H^{1}(\Delta, \Omega)$ and $v \in H^{1}(\Omega)$. Hence, for the solution of (A.1) we have

$$
\left\langle\frac{\partial y}{\partial n}, \tau v\right\rangle=\int_{\Gamma_{1}} u\left(\tau v_{\mid \Gamma_{1}}\right) d s \quad \text { for all } v \in V,
$$

which yields $\frac{\partial y}{\partial n}=u$ on $\Gamma_{1}$. This completes the proof.

\section{REFERENCES}

[1] M. Bergounioux, K. Ito, and K. Kunisch, Primal-dual strategy for constrained optimal control problems, SIAM J. Control Optim. 37, 1176-1194 (1999)

[2] M. Bergounioux and K. Kunisch, Augmented Lagrangian techniques for elliptic state constrained optimal control problems, SIAM J. Control Optim. 35, 1524-1543 (1997)

[3] D. P. Bertsekas, Projected Newton methods for optimization problems with simple constraints, SIAM J. Control Optim. 20, 221-246 (1982)

[4] M. Bergounioux, M. Haddou, M. Hintermüller, and K. Kunisch, A comparison of a Moreau-Yosidabased active set strategy and interior point methods for constrained optimal control problems, SIAM J. Optim. 11, 495-521 (2000)

[5] R. Dautray and J.-L. Lions, Mathematical analysis and numerical methods for science and technology, Vol. 2, Springer-Verlag, 1988 
[6] J. E. Dennis, M. Heinkenschloss, and L. N. Vicente, TRICE: Trust-region interior-point SQP algorithms for optimal control and engineering design problems. http://www. caam.rice.edu/ trice

[7] J. C. Dunn, On $L^{2}$ sufficient conditions and the gradient projection method for optimal control problems, SIAM J. Control Optim. 34, 1270 1290 (1996)

[8] P. Gill, W. Murray, and M. H. Wright, Practical Optimization, Academic Press, 1999 (reprint)

[9] D. Goldfarb and A. Idnani, A numerically stable dual method for solving strictly convex quadratic programs, Math. Prog. 21, 1-33 (1983)

[10] P. Grisvard, Singularities in boundary value problems, Research Notes in Applied Mathematics 22, Springer-Verlag, 1992

[11] W. W. Hager, The dual active set algorithm, Advances in Optimization and Parallel Computing, 1992, pp. 137-142

[12] W. W. Hager, Application of the dual active set algorithm to quadratic network optimization, Comput. Optim. Appl. 1. 349-373 (1993)

[13] M. Heinkenschloss, M. Ulbrich, and S. Ulbrich, Global convergence of trust-region interior-point algorithms for infinite-dimensional nonconvex minimization subject to pointwise bounds, preprint TR97-04, Dept. of Computational and Applied Mathematics, Rice University, Houston, 1997

[14] K. Ito and K. Kunisch, Augmented Lagrangian-SQP methods in Hilbert spaces and applications to control in the coefficient problems, SIAM J. Optim. 6, 96-125 (1996)

[15] K. Ito and K. Kunisch, Augmented Lagrangian methods for nonsmooth convex optimization in Hilbert spaces, Nonlinear Analysis, Theory, Methods and Applications 41, 591-616 (2000)

[16] C. T. Kelley and E. W. Sachs, Multilevel algorithms for constrained compact fixed point problems. Iterative methods in numerical linear algebra, SIAM J. Sci. Comput. (3) 15, 645-667 (1994)

[17] F. Leibfritz and E. W. Sachs, Inexact SQP interior point methods and large scale optimal control problems, preprint, University of Trier, 1995, SIAM J. Control Optim. 38, 272-293 (1999)

[18] J. L. Lions, Some aspects of the optimal control of distributed parameter systems, Regional Conference Series in Applied Mathematics 6, SIAM, 1972

[19] C. Lin and J. J. Moré, Newton's method for large bound-constrained optimization problems, preprint, Argonne National Laboratory, Mathematics and Computer Science Division, 1999; SIAM J. Optim. 9, 1100-1127 (1999)

[20] J. J. Moré and G. Toraldo, Algorithms for bound constrained quadratic programming problems, Numer. Math. 55, 377-400 (1989)

[21] J. J. Moré and G. Toraldo, On the solution of large quadratic programming problems with bound constraints, SIAM J. Optim. 1 93-113 (1991)

[22] T. Tian and J. C. Dunn, On the gradient projection method for optimal control problems with nonnegative $L^{2}$ inputs, SIAM J. Control Optim. 32, 516-537 (1994)

[23] M. Tinkham, Introduction to Superconductivity, McGraw-Hill, N.Y., 1975

[24] G. M. Troianiello, Elliptic differential equations and obstacle problems, Plenum Press, N.Y., 1987

[25] F. Tröltzsch, An SQP-method for optimal control of a nonlinear heat equation, Control Cybernet. 23, 268-288 (1994)

[26] R. J. Vanderbei, Linear programming: Foundations and extensions, Kluwer, 1997

[27] S. J. Wright, Primal-dual interior point methods, SIAM, 1997

[28] Y. Ye, Interior point algorithms: Theory and analysis, John Wiley and Sons, 1997 